CGPG-97/8-1

g-alg/9708030

\title{
QUANTIZATION OF EQUIVARIANT VECTOR BUNDLES
}

\author{
ELI HAWKINS \\ Center for Gravitational Physics and Geometry \\ The Pennsylvania State University, University Park, PA 16802 \\ E-mail: mrmuon@phys.psu.edu
}

\begin{abstract}
The quantization of vector bundles is defined. Examples are constructed for the well controlled case of equivariant vector bundles over compact coadjoint orbits. (A coadjoint orbit is a symplectic manifold with a transitive, semisimple symmetry group.) In preparation for the main result, the quantization of coadjoint orbits is discussed in detail.

This subject should not be confused with the quantization of the total space of a vector bundle such as the cotangent bundle.
\end{abstract}

\section{Contents}

1. Introduction 2

2. Quantization 4

2.1. Direct and inverse limit quantization 5

3. Quantized Vector Bundles 6

3.1. Direct and inverse limits 6

\begin{tabular}{lll}
\hline 4. & Classical Homogeneous Spaces & 7
\end{tabular}

\begin{tabular}{lll}
\hline 4.1. & The set of coadjoint orbits & 7
\end{tabular}

$\begin{array}{lll}4.2 . & \text { Equivariant bundles } & 7\end{array}$

5. Quantized Coadjoint Orbits 8

$\begin{array}{llr}\text { 5.1. Generators and relations picture } & 8\end{array}$

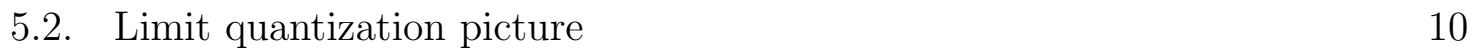

$\begin{array}{lll}5.3 . & \text { Convergence } & 12\end{array}$

$\begin{array}{lll}5.4 . & \text { Polynomials } & 13\end{array}$

\begin{tabular}{lll}
\hline 6. & Quantization of Vector Bundles over $\mathcal{O}_{\Lambda}$ & 14
\end{tabular}

$\begin{array}{llll}6.1 . & \text { General quantized bundles } & 14\end{array}$

$\begin{array}{lll}6.2 . & \text { Limit quantized bundles } & 16\end{array}$

\begin{tabular}{lll}
\hline 6.3. & Identification with bundles & 16
\end{tabular}

\begin{tabular}{lll}
\hline 6.4 . The allowed weights & 17
\end{tabular}

\begin{tabular}{ll}
\hline 7. & Further Remarks on Bundles \\
\hline
\end{tabular}

$\begin{array}{ll}7.1 . \quad \text { Uniqueness } & 17\end{array}$

\begin{tabular}{lll}
\hline 7.2. & Geometric Quantization & 18
\end{tabular}

1991 Mathematics Subject Classification. 58F06; Secondary 46L87, 58B30. 
\begin{tabular}{lll}
\hline 7.3. & Bimodules & 18
\end{tabular}

8. The Case of the 2-sphere 19

\begin{tabular}{lll}
\hline 9. & Final remarks & 21
\end{tabular}

Appendix A. Sections 22

\begin{tabular}{ll}
\hline Appendix B. Limits & 23
\end{tabular}

\begin{tabular}{|lll}
\hline B.1. Direct limit of algebras & 23
\end{tabular}

B.2. Direct limit of modules 25

\begin{tabular}{lll}
\hline B.3. & Inverse limit of algebras & 25
\end{tabular}

\begin{tabular}{|ll} 
B.4. Inverse limit of modules & 26
\end{tabular}

\begin{tabular}{|lll}
\hline Appendix C. & Review of Representation Theory & 26
\end{tabular}

\begin{tabular}{lll}
\hline Appendix D. Coadjoint Orbits & 28
\end{tabular}

\begin{tabular}{lll}
\hline D.1. Symplectic structure & 28
\end{tabular}

\begin{tabular}{lll}
\hline D.2. & Why coadjoint orbits? & 28
\end{tabular}

\begin{tabular}{lll}
\hline D.3. & Structure of coadjoint orbits & 29
\end{tabular}

D.4. Examples 30

\begin{tabular}{lll}
\hline Appendix E. & Projective space & 31
\end{tabular}

Notation $\quad 32$

\begin{tabular}{ll}
\hline Acknowledgments & 32
\end{tabular}

$\begin{array}{ll}\text { References } & 33\end{array}$

\section{INTRODUCTION}

Quantization is a vaguely defined process by which a noncommutative algebra is generated from some ordinary, commutative space. Traditionally this space has been the phase space of some system in classical mechanics; the algebra is then meant to consist of observables for a corresponding quantum system. A more recent use of quantization is with a space that is thought of geometrically; the quantization is then thought of as giving noncommutative geometries which approximate the original space being considered.

The existing theory of quantization is limited for this purpose in that it only gives an algebra. This corresponds to just having the topology of the quantized space (see (4⿴囗十). If the original space has more interesting structures than just its topology, then it would be desirable to in some sense "quantize" these as well.

Arguably, vector bundles are the most important structures beyond point set topology. Most structures used in geometry are, or involve, vector bundles. The vector fields, differential forms, and spinor fields are sections of vector bundles. $K$-theory is constructed from vector bundles. A Riemannian metric is a section of a bundle. Differential operators, such as the Dirac operator, act on sections of vector bundles. Indeed, in physics most fields are sections of vector bundles.

This paper is a first step towards a theory of the quantization of vector bundles. In pursuit of this goal, I present a plausible definition for the quantization of a vector bundle, and illustrate it with a large class of examples. I give a more general construction of quantization of vector bundles in [14]. 
I only consider compact manifolds in this paper for several reasons. One is that this is inevitably the simplest case to deal with, since almost anything that will work generally will work in the compact case. Another is physically motivated. The most natural quantizations of compact manifolds give finite-dimensional algebras; as a result, the degrees of freedom of anything on the space should become finite after quantization. This can, therefore, be used as a regularization technique for quantum field theories (see [8]). Outside of some definitions, I will assume the space is a compact manifold $\mathcal{M}$ and the quantizations are finite-dimensional.

In order to get simple examples I will assume that the geometry is also highly symmetrical. Suppose that some compact, semisimple Lie group acts transitively円 on $\mathcal{M}$, and that everything is equivariant under the action of this group. A manifold that can be quantized (to give finite-dimensional algebras) in a reasonable sense must have a symplectic structure (App. D.1). A symplectic manifold with transitive symmetry by a compact, semisimple Lie group must be equivalent to a coadjoint orbit of that group (App. D.2). The coadjoint orbits are therefore the only spaces that can be quantized nicely with this much symmetry. Luckily, coadjoint orbits of compact Lie groups have a very simple systematic quantization (Sec. 5).

I begin in Section 2 with a general definition of quantization structure similar to that given by Berezin in [1]. This definition involves a minimum of structure. However, greater structure can be useful for some purposes.

The perspective of noncommutative geometry [4] holds that a noncommutative algebra should correspond to the "true" geometry, and that the "classical limit" is merely a convenient approximation to this [3, 5]. This suggests that the classical algebra of functions should be secondary, constructed as the limit of a sequence of noncommutative algebras. Based on this philosophy (and other motivations described in Sec. 9), in Section 2.1 I outline an approach to quantization based on a directed or inverse system of algebras whose limit is the classical algebra of continuous functions; I call these structures direct and inverse limit quantizations. The technical details of these limits are discussed in Appendices B.1 and B.3.

In Section 3, I give a definition for the quantization of a vector bundle. Like the quantization of an algebra, the quantization of a vector bundle can be viewed in terms of a directed or inverse system. This is described in Section 3.1 and detailed in Appendices B.2 and B.4.

The most relevant properties of homogeneous spaces and their vector bundles are described in Section 4 . In Appendix D, I describe the reasons that the spaces considered here are all coadjoint orbits, and then discuss some properties of these spaces. Appendix D.3 describes the classification of the coadjoint orbits for a given group, and gives a diagrammatic technique for expressing a coadjoint orbit as a coset space.

The standard quantization of coadjoint orbits is reviewed, and described in perhaps new ways, in Section 5. The quantization is constructed using generators and relations in Section 5.1. The directed and inverse limit quantizations are constructed in 5.2.

\footnotetext{
${ }^{1}$ I. e., the group can take any point to any other point.
} 
Appendix $\mathbb{E}$ gives some additional details which are relevant to the discussion of convergence of the direct and inverse limit quantizations in 5.3.

Section 6 contains the main results of this paper. I first construct quantized vector bundles, and then determine what bundles these are quantizations of. I show that all equivariant vector bundles over coadjoint orbits may be quantized.

I then discuss some matters arising from this construction. In 7.1 I explain the extent to which the construction is unique. In 7.2 I note an interesting relationship to geometric quantization. In 7.3 I note a property that these quantizations fail to have.

In order to illustrate the constructions in this paper, I describe some of the details in the simplest possible case, that of $S^{2}$, in Section 8 .

Appendices $\mathrm{A}$ and $\mathrm{C}$ serve to fill in some background and fix notation. Appendix $\mathrm{A}$ is background mainly for Appendix B. Some of the relevant facts about Lie groups are reviewed in Appendix $\mathrm{Q}$ in a perspective appropriate to this paper.

This topic unfortunately requires using a great many symbols. A table of notations is provided at the end of the paper.

\section{Quantization}

Generally, quantization refers to some sort of correspondence between an algebra of functions on some space, and some noncommutative algebra. This might involve a map that identifies functions to operators in the noncommutative algebra, or perhaps vice versa. The idea of a "classical limit" is that the algebra of quantum operators becomes the algebra of classical functions in some limiting sense. To make this meaningful requires having not one, but a whole sequence (discrete or continuous) of quantum algebras.

This idea can be made more concrete. Let all algebras involved be $\mathrm{C}^{*}$-algebras. Call the space $\mathcal{M}$; the algebra of functions is the algebra $\mathcal{C}_{0}(\mathcal{M})$ of continuous functions (vanishing at infinity in the noncompact case). The set of quantum algebras may be parameterized either continuously (say, over $\mathcal{I}=\mathbb{R}_{+}$) or discretely (say, over $\mathcal{I}=\mathbb{N}$ ). Compactify the parameter space $\mathcal{I}$ by adjoining some " $\infty$ " where the classical limit belongs. The algebras form a bundle $\mathcal{A}_{\hat{\mathcal{I}}}$ over this completed parameter space $\hat{\mathcal{I}}=\mathcal{I} \cup\{\infty\}$, each quantum algebra is the fiber over its parameter and $\mathcal{C}_{0}(\mathcal{M})$ is the fiber over $\infty$. This $\mathcal{A}_{\hat{\mathcal{I}}}$ should in fact be a continuous field of $\mathrm{C}^{*}$-algebras; see [6].

I am taking the perspective in this paper that quantization gives noncommutative approximations to the topology $\mathcal{M}$. From this perspective, the most essential information about the quantum-classical correspondence is encoded in the topology of the bundle $\mathcal{A}_{\hat{\mathcal{I}}}$. A sequence of operators in each of the quantum algebras can be reasonably identified with a certain function only if these together form a continuous section of $\mathcal{A}_{\hat{\mathcal{I}}}$. The space of continuous sections over $\hat{\mathcal{I}}$ is naturally a $\mathrm{C}^{*}$-algebra, $\mathbb{A}:=\Gamma\left(\mathcal{A}_{\hat{\mathcal{I}}}\right)$ (see App. A). There is a natural surjection $\mathcal{P}: \mathbb{A} \rightarrow \mathcal{C}_{0}(\mathcal{M})$ which is simply eval-

uation at the point $\infty \in \hat{\mathcal{I}}$. This algebra and surjection are the most succinct and bare-bones quantization structure. This will be referred to as a general quantization. 
This is almost the same as the structure of quantization given by Berezin in [1] It is also a generalization of the structure of a strict deformation quantization [19]; in that case the index set $\hat{\mathcal{I}}$ is required to be an interval.

Other quantization structures contain more (possibly irrelevant) information. Suppose that we are given a quantization of a space $\mathcal{M}$ in the form of a sequence of algebras $\left\{\mathcal{A}_{N}\right\}_{N=1}^{\infty}$ and maps $P_{N}: \mathcal{C}_{0}(\mathcal{M}) \rightarrow \mathcal{A}_{N}$. This is a pretty typical quantization structure; the operator $P_{N}(f)$ is considered to be the quantization of the function $f$. The topology I give to $\mathcal{A}_{\hat{\mathbb{N}}}=\mathcal{A}_{\mathbb{N}} \cup \mathcal{C}(\mathcal{M})$ is the weakest such that for each $f \in \mathcal{C}_{0}(\mathcal{M})$ the section taking $N \mapsto P_{N}(f)$ and $\infty \mapsto f$ is continuous. Two sets of $P_{N}$ 's that give the same topology to the bundle are equivalent for the purposes of my perspective.

This structure of general quantization is not tied to any particular method of quantization. Indeed, it need not correspond to something that would usually be called quantization. The point of it is that a large class of concepts of quantization can be used to construct a general quantization structure, and it is this structure which is relevant to defining the quantization of a vector bundle in Section 3 .

The strategy for constructing general quantizations that is used here is that $\mathbb{A} \equiv$ $\Gamma\left(\mathcal{A}_{\hat{\mathcal{I}}}\right)$ is a subalgebra of $\Gamma_{\mathrm{b}}\left(\mathcal{A}_{\mathcal{I}}\right)$ (the $\mathrm{C}^{*}$-algebra of bounded sections over $\mathcal{I}$; see App. A). The difference between these two types of sections is the behavior approaching $\infty$; elements of $\Gamma\left(\mathcal{A}_{\hat{\mathcal{I}}}\right)$ must be continuous at $\infty$. The key is to describe the condition of continuity at $\infty$ purely in terms of $\mathcal{I} \not \supset \infty$.

2.1. Direct and inverse limit quantization. In this section I make the assumption that $\mathcal{M}$ is compact and the quantum algebras are finite-dimensional. Since dimensions change discretely, the simplest choice of parameter space is $\mathcal{I}=\mathbb{N}$.

One perspective on quantization is that the classical algebra is literally the limit of the sequence of quantum algebras. A limit of algebraic objects is generally constructed from either a "directed system" or "inverse system", so those are what I use here. The former is a bundle of algebras $\mathcal{A}_{\mathbb{N}}$ and a sequence of maps $i_{N}: \mathcal{A}_{N} \hookrightarrow \mathcal{A}_{N+1}$ linking them together. In the latter the maps are in the opposite direction, $p_{N}: \mathcal{A}_{N} \rightarrow \mathcal{A}_{N-1}$. If constructed properly, these types of systems have limits $\lim \left\{\mathcal{A}_{*}, i_{*}\right\}$ and $\lim _{\longleftarrow}\left\{\mathcal{A}_{*}, p_{*}\right\}$ which are $\mathrm{C}^{*}$-algebras; these are detailed in Appendices B.1 and B.3.

Intuitively, the directed system can be thought of as

$$
\mathcal{A}_{1} \stackrel{i_{1}}{\longleftrightarrow} \mathcal{A}_{2} \stackrel{i_{2}}{\longrightarrow} \ldots \longleftrightarrow \lim _{\longrightarrow}\left\{\mathcal{A}_{*}, i_{*}\right\} .
$$

For every $N$ there is a composed injection $I_{N}: \mathcal{A}_{N} \hookrightarrow \underline{\lim }\left\{\mathcal{A}_{*}, i_{*}\right\}$. These satisfy a consistency condition with the $i_{N}$ 's that $I_{N}=I_{N+1} \circ i_{N}$. Similarly, the inverse system can be thought of as

$$
\mathcal{A}_{1} \stackrel{p_{2}}{\longleftarrow} \mathcal{A}_{2} \stackrel{p_{3}}{\longleftarrow} \ldots \longleftarrow \lim \left\{\mathcal{A}_{*}, p_{*}\right\} .
$$

There are composed surjections $P_{N}: \lim \left\{\mathcal{A}_{*}, p_{*}\right\} \rightarrow \mathcal{A}_{N}$. These also satisfy a consistency condition that $P_{N}=p_{N+1} \circ P_{N+1}$. These $I_{N}$ 's and $P_{N}$ 's are part of the general

\footnotetext{
${ }^{2}$ The major difference is that Berezin used smooth rather than continuous functions.
} 
constructions of directed and inverse limits. The general quantization algebra $\mathbb{A}$ is also a natural byproduct of these constructions.

The maps $i_{N}$ and $p_{N}$ used in these must not be assumed to be (multiplicative) homomorphisms in general. That assumption would actually restrict $\mathcal{M}$ to be a totally disconnected space, which is almost certainly not what we want. Instead we must allow these maps to be some more general type of morphisms, such as unital completely positive maps 3 This is discussed a little more in Appendix B.1.

\section{Quantized Vector Bundles}

Suppose that we are given a finitely generated vector bundle $V \rightarrow \mathcal{M}$ (see [20]). If the algebra of functions $\mathcal{C}_{0}(\mathcal{M})$ is quantized, then what should be meant by the quantization of $V$ ? In noncommutative geometry, all geometrical structures are dealt with algebraically. In order to find the appropriate definition for quantization of $V$, we must first treat $V$ algebraically. The algebraic approach comes from the fact that the continuous sections $\Gamma_{0}(V)$ form a finitely generated, projective module of the algebra $\mathcal{C}_{0}(\mathcal{M})$. Indeed, this gives a one-to-one correspondence between finitely generated, locally trivial, vector bundles and finitely generated, projective modules (see [4]). The "quantization" of $V$ should give modules for each of the quantum algebras $\mathcal{A}_{N}$; in other words, a bundle of modules over $\mathcal{I}$.

I define a quantization of the bundle $V$ to be a bundle of modules $V_{\hat{\mathcal{I}}}$ over $\hat{\mathcal{I}}$ such that the topology is consistent with that of $\mathcal{A}_{\hat{\mathcal{I}}}$, and the fiber at $\infty$ is the module $\Gamma_{0}(V)$.

The space of sections $\mathbb{V}:=\Gamma\left(V_{\hat{\mathcal{I}}}\right)$ is a module of $\mathbb{A}$. This gives another way of describing the quantization of $V$. A quantization of $V$ may be equivalently defined as a finitely generated, projective module $\mathbb{V}$ of $\mathbb{A}$ satisfying the sole condition that the push-forward by $\mathcal{P}$ to a module of $\mathcal{C}_{0}(\mathcal{M})$ is $\Gamma_{0}(V)$. The condition that $\mathcal{A}_{\hat{\mathcal{I}}}$ and $V_{\hat{\mathcal{I}}}$ have consistent topologies is implicitly encoded in this definition.

Just as a continuous function is not uniquely determined by its value at a single point, there is not a single, unique quantization of a given $V$. Indeed, when $\mathcal{I}$ is discrete, any finite subset of $V_{N}$ 's can be changed arbitrarily. However, there may be a uniquely natural choice for almost all $V_{N}$ 's given by a single formula. This is so in the case discussed in this paper. This issue is discussed further in Section 7.1.

The guiding principle for quantizing vector bundles will be that we already have one example. The sections of the trivial line bundle $V=\mathcal{M} \times \mathbb{C}$ are simply the continuous functions $\mathcal{C}_{0}(\mathcal{M})$. This means that $\mathbb{V}=\mathbb{A}$ should always be a good quantization of this bundle.

3.1. Direct and inverse limits. Return to the assumptions of Section 2.1 (compactness, etc.). As with quantizing $\mathcal{C}(\mathcal{M})$, it is possible to use additional structure in the quantization of a vector bundle. A quantized vector bundle can be constructed from a directed system $\left\{V_{*}, \iota_{*}\right\}$ or an inverse system $\left\{V_{*}, \pi_{*}\right\}$ of modules. In these

\footnotetext{
${ }^{3}$ The property of complete positivity will not be used here; although it will be mentioned several times. For definition and discussion see [16].
} 
systems, each $V_{N}$ is an $\mathcal{A}_{N}$-module; the maps are linear maps $\iota_{N}: V_{N} \hookrightarrow V_{N+1}$ and $\pi_{N}: V_{N} \rightarrow V_{N-1}$. The details of this are described in Appendices B.2 and B.4. There are again composed injections $I_{N}^{V}$ and surjections $P_{N}^{V}$, satisfying the same sort of compatibility conditions as for $I_{N}$ and $P_{N}$ in Section 2.1.

\section{Classical Homogeneous Spaces}

Again, and throughout the rest of this paper, I assume that $\mathcal{M}$ is a compact manifold, the parameter space is $\mathcal{I}=\mathbb{N}$, and the algebras $\mathcal{A}_{N}$ are finite-dimensional. In order to get some control of the system, and construct some quantizations explicitly, let us assume that some group $G$ acts transitively on $\mathcal{M}$ (i. e., $\mathcal{M}$ is homogeneous) and that everything we do will be $G$-equivariant. It is a standard construction (see [15]) that $\mathcal{M}$ can be written as a coset space $\mathcal{M}=G / H$ where the isotropy group is $H:=\{h \in G \mid h(o)=o\}$ for some arbitrary basepoint $o \in \mathcal{M}$. Since $\mathcal{M}$ is a manifold, $G$ is best chosen to be a Lie group. If we assume $G$ to be compact and semisimplef then the set of $\mathcal{M}$ 's we are interested in is (up to equivalence) the set of "coadjoint orbits" (see App. D.2).

4.1. The set of coadjoint orbits. The coadjoint space is $\mathfrak{g}^{*}$, the linear dual of the Lie algebra $\mathfrak{g}$ of $G$. There is a natural, linear action of $G$ on $\mathfrak{g}^{*}$. A coadjoint orbit is simply the orbit of some point in $\mathfrak{g}^{*}$ under that $G$ action.

The relevant definitions concerning Lie groups are summarized in Appendix $\mathrm{C}$. The classification of coadjoint orbits is strikingly similar to the classification of irreducible representations. The irreducible representations are classified by the dominant weights, which are the vectors on the weight lattice that lie in the positive Weyl chamber $\mathcal{C}_{+} \subset \mathfrak{g}^{*}$. The coadjoint orbits are classified by all vectors in $\mathcal{C}_{+}$(see App. D.3). Denote by $\mathcal{O}_{\Lambda}$ the coadjoint orbit of $\Lambda \in \mathcal{C}_{+} \subset \mathfrak{g}^{*}$.

Since a coadjoint orbit is a homogeneous space, it can always be expressed as a coset space $\mathcal{O}_{\Lambda} \cong G / H$; it is natural to identify the basepoint $o=e H \in G / H$ with $\Lambda \in \mathcal{O}_{\Lambda}$. A diagrammatic method of calculating $H$ from $\Lambda$ is described in Appendix D.3.

The structures of the sets of irreducible representations of $G$ and of $H$ are closely related. The weight lattices of $G$ and $H$ are naturally identified. However, the sets of weights which are dominant (and thus actually correspond to representations) are different. This is relevant in Section 6.3.

\subsection{Equivariant bundles.}

Notation. In this paper I will generally refer to a representation space (group module) simply as a representation.

Suppose that $V$ is an equivariant vector bundle over $\mathcal{M}=G / H$. This simply means that $\Gamma(V)$ is a representation of $G$. The fiber $V_{o}$ at the basepoint $o=e H$ is a vector space and is acted on by $H$, so $V_{o}$ is a representation of $H$.

\footnotetext{
${ }^{4}$ Assuming $G$ semisimple is equivalent to assuming $\mathcal{M}$ is not a torus or the product of a torus with something else.
} 
Suppose that $W$ is a representation of $H$. The set $W \times_{H} G:=W \times G / \sim$, where $(w, g) \sim\left(h w, g h^{-1}\right)$, is naturally an equivariant vector bundle over $\mathcal{M}$. The bundle surjection $W \times_{H} G \rightarrow G / H$ is $[(w, g)] \mapsto g H$; the action of $g^{\prime} \in G$ is $[(w, g)] \mapsto$ $\left[\left(w, g^{\prime} g\right)\right]$. Up to equivalence, all equivariant vector bundles may be constructed in this way.

The fiber of $W \times_{H} G$ at $o$ is simply $W$, so there is a one-to-one correspondence between $H$-representations and equivariant vector bundles over $\mathcal{M}$. The semigroup of equivariant vector bundles under direct sum is generated by the set of irreducible bundles — those corresponding to irreducible representations.

It is not the case that all vector bundles over $\mathcal{M}$ can be made equivariant. Nevertheless, I am only considering equivariant bundles in this paper. Every bundle over a homogeneous space which is mentioned in this paper is a finitely generated, locally trivial, equivariant, vector bundle; but I will frequently omit some of these adjectives.

\section{Quantized Coadjoint Orbits}

Notation. The irreducible representations of $G$ are in one-to-one correspondence with dominant weights (App. C). Denote the space of the representation corresponding to the weight $\lambda$ by $(\lambda)$. This is the $G$-representation with "highest weight" $\lambda$ (App. C).

Denote $\mathcal{A}_{N}:=\operatorname{End}(N \Lambda)$, the algebra of matrices on the vector space $(N \Lambda)$; the notation $\mathcal{A}_{N}$ will be justified in the following.

5.1. Generators and relations picture. The action of $\mathfrak{g}$ on $(N \Lambda)$ can be expressed as a map $\mathfrak{g} \rightarrow \operatorname{End}(N \Lambda)=\mathcal{A}_{N}$. The associative algebra $\mathcal{A}_{N}$ is generated by the image of the Lie algebra $\mathfrak{g}$. Let $\left\{J_{i}\right\} \subset \mathfrak{g}$ be a basis of self-adjoint generators of $\mathfrak{g}$ acting on $(N \Lambda) ; \mathcal{A}_{N}$ can be written in terms of this set of generators and the following relations.

First, the commutation relations state that

$$
\left[J_{i}, J_{j}\right]_{-}=i C_{i j}^{k} J_{k}
$$

where $C_{i j}^{k}$ are the structure coefficients. Second, the Casimir relations state that

$$
\mathcal{C}_{n}(J)=c_{n}(N \Lambda) \quad \forall n,
$$

where the Casimirs $\mathcal{C}_{n}$ are $G$-invariant, symmetrically ordered, homogeneous polynomials in the $J$ 's, and the $c_{n}$ 's are the corresponding eigenvalues. Finally, the Serre relations state that certain linear combinations of $J_{i}$ 's are nilpotent, the order of nilpotency rising linearly with $N$; an example of this is given in Section 8 .

The Casimir eigenvalues $c_{n}(N \Lambda)$ are polynomials in $N \Lambda$ of the same order as $\mathcal{C}_{n}$. In fact the leading order (in $N$ ) term is $\mathcal{C}_{n}(\Lambda) N^{\operatorname{Ord}\left(\mathcal{C}_{n}\right)}$. The reason that it is meaningful to evaluate $\mathcal{C}_{n}$ on a point of $\mathfrak{g}^{*}\left(\right.$ such as $\Lambda$ ) as well as on the $J_{i}$ 's is that the $J_{i}$ 's together form a sort of Lie algebra valued vector in $\mathfrak{g}^{*}$.

The Serre relations are actually equivalent to the condition that the $J_{i}$ 's generate a $\mathrm{C}^{*}$-algebra. Suppose that the $J_{i}$ 's do lie inside a $\mathrm{C}^{*}$-algebra and satisfy the commutation and Casimir relations. Then this $\mathrm{C}^{*}$-algebra can be faithfully represented on a Hilbert space $\mathcal{H}$. The commutation relations imply that the $J_{i}$ 's generate a unitary 
representation of $G$ on $\mathcal{H}$. The Casimir relations imply that $\mathcal{H}$ can only be $(N \Lambda)$ or some Hilbert space direct sum of copies of $(N \Lambda)$. This means that the $\mathrm{C}^{*}$-subalgebra generated by the $J_{i}$ 's is $\operatorname{End}(N \Lambda)$; which implies that the Serre relations are satisfied.

Now, regard the $\mathcal{A}_{N}$ 's as forming a bundle $\mathcal{A}_{\mathbb{N}}$ over the discrete parameter space $\mathbb{N}$. We can think of $N$ and the generators $J_{i}$ as sections in $\Gamma\left(\mathcal{A}_{\mathbb{N}}\right)$, but neither is bounded, so they are not in $\Gamma_{\mathrm{b}}\left(\mathcal{A}_{\mathbb{N}}\right)$ (the $\mathrm{C}^{*}$-algebra of bounded sections; see App. A). However, the combinations $X_{i}=N^{-1} J_{i}$ are bounded; as can be seen by considering the quadratic Casimir $\mathcal{C}_{1}$. This means that $X_{i} \in \Gamma_{\mathrm{b}}\left(\mathcal{A}_{\mathbb{N}}\right)$.

Define $\mathbb{A}$ to be the $\mathrm{C}^{*}$-subalgebra of $\Gamma_{\mathrm{b}}\left(\mathbb{A}_{\mathbb{N}}\right)$ generated by the $X_{i}$ 's. Define $\mathbb{A}_{0}:=$ $\Gamma_{0}\left(\mathcal{A}_{\mathbb{N}}\right)$ to be the algebra of sections vanishing at $\infty$ (see App. A); since in fact $\mathbb{A}_{0}$ is contained in $\mathbb{A}$, it is an ideal there Define

$$
\mathcal{P}: \mathbb{A} \rightarrow \mathcal{A}_{\infty}:=\mathbb{A} / \mathbb{A}_{0}
$$

to be the corresponding quotient homomorphism; this essentially just evaluates the $N \rightarrow \infty$ limit.

By construction, the images $x_{i}:=\mathcal{P}\left(X_{i}\right)$ generate the quotient algebra $\mathcal{A}_{\infty}$. The relations these satisfy all derive from the relations satisfied by the $X_{i}$ 's. These generators commute, since

$$
\left[x_{i}, x_{j}\right]_{-}=\mathcal{P}\left(\left[X_{i}, X_{j}\right]_{-}\right)=\mathcal{P}\left(i N^{-1} C_{i j}^{k} X_{k}\right)=0,
$$

so $\mathcal{A}_{\infty}$ is a commutative $\mathrm{C}^{*}$-algebra (and therefore is the algebra of continuous functions on some space). The $x_{i}$ 's transform under $G$ in the same way as Cartesian coordinates on $\mathfrak{g}^{*}$, so $\mathcal{A}_{\infty}$ is the algebra of continuous functions on some subspace of $\mathfrak{g}^{*}$. The non-Serre relations alone define a $\mathrm{C}^{*}$-algebra; therefore the Serre relations do not give any additional relations for $\mathcal{A}_{\infty}$. The only other relations the $x_{i}$ 's satisfy are polynomial relations

$$
\mathcal{C}_{n}(x) \stackrel{!}{=} \lim _{N \rightarrow \infty} N^{-\operatorname{Ord}\left(\mathcal{C}_{n}\right)} c_{n}(N \Lambda)=\mathcal{C}_{n}(\Lambda)
$$

which make $\mathcal{A}_{\infty}$ the algebra of continuous functions on the algebraic subspace $\mathcal{M} \subset \mathfrak{g}^{*}$ determined by these polynomials.

The Casimir polynomials are a complete system of $G$-invariant polynomials; therefore $\mathcal{M}$ must be a single coadjoint orbit. Obviously, $x=\Lambda$ satisfies $\mathcal{C}_{n}(x)=\mathcal{C}_{n}(\Lambda)$, so $\Lambda \in \mathcal{M}$; therefore $\mathcal{M}$ is the orbit $\mathcal{O}_{\Lambda}$. This shows that in the sense of Section 2, the system $\mathcal{P}: \mathbb{A} \rightarrow \mathcal{C}\left(\mathcal{O}_{\Lambda}\right)$ is a general quantization of $\mathcal{O}_{\Lambda}$.

In this construction the $\Lambda$ was required to be integral (a weight) rather than any arbitrary $\Lambda \in \mathcal{C}_{+}$. However, this is not a serious restriction. Rescaling $\Lambda$ simply rescales $\mathcal{O}_{\Lambda}$, therefore a more appropriate parameter space for distinct coadjoint orbits is the projectivisation $\mathbb{P C _ { + }}$. The image of the weights is dense in $\mathbb{P} \mathcal{C}_{+}$(it is the set

\footnotetext{
${ }^{5}$ The eigenvalue of the quadratic $\mathcal{C}_{1}(X)$ is $\mathcal{C}_{1}(\Lambda)$ plus a term proportional to $N^{-1}$, therefore it is bounded as $N \rightarrow \infty$, therefore it is a polynomial of bounded operators.

${ }^{6}$ It is essentially sufficient to show that $\mathbb{A}$ contains one function on $\mathbb{N}$ that nontrivially converges to 0 .

${ }^{7}$ Because $\mathbb{A}_{0}$ is an ideal in $\Gamma_{\mathrm{b}}\left(\mathbb{A}_{\mathbb{N}}\right)$.
} 
of "rational" points), so the quantizable coadjoint orbits are dense in the space of distinct coadjoint orbits.

\subsection{Limit quantization picture.}

Notation. The linear dual of an irreducible representation is also an irreducible representation; we can therefore define $\lambda^{*}$ by the property $\left(\lambda^{*}\right)=(\lambda)^{*}$. This is a linear transformation on the weights (see App. C). With this notation $\mathcal{A}_{N} \equiv \operatorname{End}(N \Lambda)=$ $(N \Lambda) \otimes\left(N \Lambda^{*}\right)$.

Given a choice of Cartan subalgebra and positive Weyl chamber, there is a preferred, 1-dimensional "highest weight subspace" in $(N \Lambda)$; choose a normalized basis vector $\Psi^{N \Lambda}$ there and call it the highest weight vector (see App. Q).

Not only do the coadjoint orbits have equivariant general quantizations, but they also admit equivariant direct and inverse limit quantizations. There are standard constructions of maps $\mathcal{A}_{N} \hookrightarrow \mathcal{C}\left(\mathcal{O}_{\Lambda}\right)$ and $\mathcal{C}\left(\mathcal{O}_{\Lambda}\right) \rightarrow \mathcal{A}_{N}$ which are suitable to be used as $I_{N}$ and $P_{N}$. I present these first.

We need an equivariant, linear injection $I_{N}: \mathcal{A}_{N} \hookrightarrow \mathcal{C}\left(\mathcal{O}_{\Lambda}\right)$. If we have such an $I_{N}$, then for every point $x \in \mathcal{O}_{\Lambda}$, evaluation at $x$ determines a linear function

$$
I_{N}(\cdot)(x): \mathcal{A}_{N} \rightarrow \mathbb{C}
$$

in other words, $x$ gives an element of the dual $\mathcal{A}_{N}^{*}$. Such an $I_{N}$ is in fact equivalent to an injection $I_{N}^{*}: \mathcal{O}_{\Lambda} \hookrightarrow \mathcal{A}_{N}^{*}=\left(N \Lambda^{*}\right) \otimes(N \Lambda)$. Since $I_{N}^{*}$ must be equivariant, it is completely specified by the image of the basepoint $o=e H$. This image must be $H$-invariant.

The highest weight vector $\Psi^{N \Lambda} \in(N \Lambda)$ is $H$-invariant, modulo phase. Its conjugate vector $\Psi^{-N \Lambda} \in\left(N \Lambda^{*}\right)$ transforms by the opposite phase, so the product $\Psi^{-N \Lambda} \otimes \Psi^{N \Lambda} \in$ $\mathfrak{g}^{*}$ is $H$-invariant. In fact, $H$ is the largest subgroup that this is invariant under. Define the image of the basepoint to be $I_{N}^{*}(o):=\Psi^{-N \Lambda} \otimes \Psi^{N \Lambda} \in \mathfrak{g}^{*}$. With this choice, $I_{N}$ is given by

$$
I_{N}(a)(g H)=\left\langle g \Psi^{N \Lambda}|a| g \Psi^{N \Lambda}\right\rangle
$$

for any $g H \in \mathcal{O}_{\Lambda}$.

There is some apparent arbitrariness in this construction. There were choices made of Cartan subalgebra, positive Weyl chamber, and phase of the highest weight vector. However, the resulting $I_{N}$ is only arbitrary by the freedom to rotate $\mathcal{O}_{\Lambda}$ about $o$ (by $H)$, and this freedom was inevitable.

We now need to construct injections $i_{N}: \mathcal{A}_{N} \hookrightarrow \mathcal{A}_{N+1}$. The question is how to get from something acting on $(N \Lambda)$ to something acting on $([N+1] \Lambda)$. The key is that precisely one copy of $([N+1] \Lambda)$ always occurs as a subrepresentation of $(\Lambda) \otimes(N \Lambda)$ (see App. C). There is a unique, natural projection

$$
\Pi_{+} \in \operatorname{Hom}_{G}[(\Lambda) \otimes(N \Lambda),([N+1] \Lambda)]
$$

which maps a vector in $(\Lambda) \otimes(N \Lambda)$ to its component in the irreducible subrepresentation $([N+1] \Lambda) \subset(\Lambda) \otimes(N \Lambda)$. Using $\Pi_{+}$, an element $A \in \operatorname{End}[(\Lambda) \otimes(N \Lambda)]$ can be 
mapped to $\Pi_{+} A \Pi_{+}^{*} \in \mathcal{A}_{N+1}$. Now, that algebra is

$$
\operatorname{End}[(\Lambda) \otimes(N \Lambda)]=\operatorname{End}(\Lambda) \otimes \operatorname{End}(N \Lambda)=\mathcal{A}_{1} \otimes \mathcal{A}_{N}
$$

There is a very simple map $\mathcal{A}_{N} \hookrightarrow \mathcal{A}_{1} \otimes \mathcal{A}_{N}$ taking $a \mapsto 1 \otimes a$. Composing these gives, as desired, a map $i_{N}: \mathcal{A}_{N} \hookrightarrow \mathcal{A}_{N+1}$ by the formula

$$
i_{N}(a)=\Pi_{+}(1 \otimes a) \Pi_{+}^{*} \text {. }
$$

This (and any map that can be written in this form) is a completely positive map (see [16]).

To verify that our $i_{N}$ really satisfies the consistency condition $I_{N+1} \circ i_{N}=I_{N}$, it is sufficient to check this at the basepoint $o \in \mathcal{O}_{\Lambda}$. So, $\forall a \in \mathcal{A}_{N}$

$$
\begin{aligned}
I_{N+1} \circ i_{N}(a)(o) & =\left\langle\Psi^{(N+1) \Lambda}\left|i_{N}(a)\right| \Psi^{(N+1) \Lambda}\right\rangle \\
& =\left\langle\Psi^{(N+1) \Lambda}\left|\Pi_{+}(1 \otimes a) \Pi_{+}^{*}\right| \Psi^{(N+1) \Lambda}\right\rangle \\
& =\left\langle\Psi^{1} \otimes \Psi^{N \Lambda}|(1 \otimes a)| \Psi^{1} \otimes \Psi^{N \Lambda}\right\rangle \\
& =\left\langle\Psi^{N \Lambda}|a| \Psi^{N \Lambda}\right\rangle=I_{N}(a)(o)
\end{aligned}
$$

and it is consistent.

The surjections come about similarly. There is a related function $e_{N}$ taking $\mathcal{O}_{\Lambda}$ to projections in $\mathcal{A}_{N}$. This maps $e_{N}: o \mapsto\left|\Psi^{N \Lambda}\right\rangle\left\langle\Psi^{N \Lambda}\right|$. Using $e_{N}$, the injection $I_{N}$ can be written as

$$
I_{N}(a)(x)=\operatorname{tr}\left[a e_{N}(x)\right]
$$

and the surjection $P_{N}$ is defined as

$$
P_{N}(f)=\operatorname{dim}(N \Lambda) \int_{\mathcal{O}_{\Lambda}} f e_{N} \epsilon
$$

where $\epsilon$ is an invariant volume form normalized to give $\mathcal{O}_{\Lambda}$ volume 1 . This map is unital and positive. It is actually the adjoint of the map $I_{N}$ if we put natural inner products on $\mathcal{A}_{N}$ and $\mathcal{C}\left(\mathcal{O}_{\Lambda}\right)$. The inner product on $\mathcal{A}_{N}$ is $\langle a, b\rangle=\widetilde{\operatorname{tr}}_{(N \Lambda)}\left(a^{*} b\right)$; where $\widetilde{\operatorname{tr}}_{(N \Lambda)}$ is the trace over $(N \Lambda)$, normalized to give $\widetilde{\operatorname{tr}}_{(N \Lambda)} 1=1$. The inner product on $\mathcal{C}(\mathcal{M})$ is $\left\langle f_{1}, f_{2}\right\rangle=\int_{\mathcal{O}_{\Lambda}} f_{1}^{*} f_{2} \epsilon$.

We will automatically satisfy the consistency with the $P_{N}$ 's if we choose $p_{N}$ to be the adjoint of $i_{N-1}$. The immediately obtained formula is

$$
p_{N}(a)=\left[\widetilde{\operatorname{tr}}_{(\Lambda)} \otimes \operatorname{id}_{\mathcal{A}_{N-1}}\right](a \oplus 0) ;
$$

where this is a partial trace of the action of $a$ on $(N \Lambda) \subset(\Lambda) \otimes([N-1] \Lambda)$. This can actually be written in essentially the same form as the $i_{N}$ 's. Precisely one copy of $([N-1] \Lambda)$ always occurs as a subrepresentation of $\left(\Lambda^{*}\right) \otimes(N \Lambda)$, so there is a corresponding projection $\Pi_{-} \in \operatorname{Hom}_{G}\left[\left(\Lambda^{*}\right) \otimes(N \Lambda),([N-1] \Lambda)\right]$. With this, define $p_{N}: \mathcal{A}_{N} \rightarrow \mathcal{A}_{N-1}$ by

$$
p_{N}(a)=\Pi_{-}(1 \otimes a) \Pi_{-}^{*} .
$$

To see that this is equivalent to (5.9a), it is sufficient to check that these agree for $a=e_{N}(o)=\left|\Psi^{N \Lambda}\right\rangle\left\langle\Psi^{N \Lambda}\right|$. These $p_{N}$ 's are also completely positive. 
5.3. Convergence. I will now show that these direct and inverse limit quantizations are both convergent by considering the "product" $I_{N}\left[P_{N}\left(f_{1}\right) P_{N}\left(f_{2}\right)\right]$ for any two functions $f_{1}, f_{2} \in \mathcal{C}\left(\mathcal{O}_{\Lambda}\right)$. This is not an associative product (compare Eq. (D.1)), since $P_{N} \circ I_{N} \neq \mathrm{id}$, but as $N \rightarrow \infty$ it nevertheless converges to the product of functions.

This "product" can be written in terms of an integration kernel as

$$
I_{N}\left[P_{N}\left(f_{1}\right) P_{N}\left(f_{2}\right)\right](x)=\iint_{\mathcal{O}_{\Lambda}} K_{N}(x, y, z) f_{1}(y) f_{2}(z) \epsilon_{y} \epsilon_{z} .
$$

The volume form $\epsilon$ is again the $G$-invariant volume form giving $\mathcal{O}_{\Lambda}$ total volume 1 . From the construction of the maps $I_{N}$ and $P_{N}$ in (5.7) and (5.8) it is immediate that

$$
K_{N}(x, y, z)=[\operatorname{dim}(N \Lambda)]^{2} \operatorname{tr}\left[e_{N}(x) e_{N}(y) e_{N}(z)\right] .
$$

If we use the identification $\mathcal{O}_{\Lambda}=G / H$, this can be factorized as

$$
K_{N}\left(g H, g^{\prime} H, g^{\prime \prime} H\right)=[\operatorname{dim}(N \Lambda)]^{2}\left\langle g \Psi^{N \Lambda} \mid g^{\prime} \Psi^{N \Lambda}\right\rangle\left\langle g^{\prime} \Psi^{N \Lambda} \mid g^{\prime \prime} \Psi^{N \Lambda}\right\rangle\left\langle g^{\prime \prime} \Psi^{N \Lambda} \mid g \Psi^{N \Lambda}\right\rangle .
$$

The factor of $[\operatorname{dim}(N \Lambda)]^{2}$ serves to normalize $K_{N}$ so that

$$
I_{N}\left[P_{N}(1) P_{N}(1)\right]=1 \text {, }
$$

as it should be since $P_{N}(1)=1$ and $I_{N}(1)=1$.

The inner products in (5.12) have several nice properties. By construction, these are certainly smooth functions. The absolute value $\left|\left\langle g \Psi^{N \Lambda} \mid g^{\prime} \Psi^{N \Lambda}\right\rangle\right|$ only depends on the points $g H, g^{\prime} H \in \mathcal{O}_{\Lambda}$, and is equal to 1 for $g H=g^{\prime} H$; but for any $g H \neq g^{\prime} H$,

$$
\left|\left\langle g \Psi^{N \Lambda} \mid g^{\prime} \Psi^{N \Lambda}\right\rangle\right|<1 \text {. }
$$

The fact that (see App. Q) $\Psi^{N \Lambda}=\Psi^{\Lambda} \otimes \cdots \otimes \Psi^{\Lambda}$, gives the convenient identity

$$
\left\langle g \Psi^{N \Lambda} \mid g^{\prime} \Psi^{N \Lambda}\right\rangle=\left[\left\langle g \Psi^{\Lambda} \mid g^{\prime} \Psi^{\Lambda}\right\rangle\right]^{N}
$$

These properties imply that for any $g H \neq g^{\prime} H$,

$$
\left\langle g \Psi^{N \Lambda} \mid g^{\prime} \Psi^{N \Lambda}\right\rangle \underset{N \rightarrow \infty}{\longrightarrow} 0
$$

exponentially. The factor $[\operatorname{dim}(N \Lambda)]^{2}$ only increases polynomially; therefore, outside any neighborhood of $x=y=z, K_{N}(x, y, z)$ vanishes uniformly as $N \rightarrow \infty$. This means that in order to investigate the $N \rightarrow \infty$ limit, it is sufficient to consider $x, y$, and $z$ close together.

Since $\mathcal{O}_{\Lambda}$ is homogeneous, we can let $x=o$ without loss of generality. In order to construct an approximation for $K_{N}$ near $o$, we need a coordinate patch about $o$. Coadjoint orbits are always Kähler manifolds, so complex coordinates are convenient. The (real) tangent fiber $T_{o} \mathcal{O}_{\Lambda}$ is naturally a complex Hermitean space and in fact can be identified to a subspace of $(\Lambda)$ which is orthogonal to $\Psi^{\Lambda}$. A suitable complex coordinate patch can be constructed by using this identification along with the exponential map; thus a neighborhood of $o$ is coordinatised by vectors in a subspace of $(\Lambda)$. Let $v$ and $\zeta$ be the complex coordinates of $y$ and $z$ respectively. Using these coordinates, to second order

$$
[\operatorname{dim}(\Lambda)]^{-2} K_{1}(o, y, z) \approx 1-\|v\|^{2}-\|\zeta\|^{2}+\langle v \mid \zeta\rangle
$$


(see App. E). A formula for $K_{N}$ (with $N \gg 1$ ) can be constructed by raising this to the $N$ 'th power and recalling the normalization (5.13). This gives

$$
K_{N}(o, y, z) \epsilon_{y} \epsilon_{z} \approx\left(\frac{N}{\pi}\right)^{2 n} e^{-N\left[\|v\|^{2}+\|\zeta\|^{2}-\langle v \mid \zeta\rangle\right]} d^{2 n} v d^{2 n} \zeta
$$

where $2 n=\operatorname{dim} \mathcal{O}_{\Lambda}$. The $L^{1}$ norm of the error in this expression is of order $N^{-\frac{3}{2}}$ and thus goes to 0 as $N \rightarrow \infty$. It is a standard result that as $N \rightarrow \infty$ a complex Gaussian such as (5.15) converges as a $\mathcal{C}^{-\infty}$ distribution to the delta distribution $\delta^{2 n}(v) \delta^{2 n}(\zeta) d^{2 n} v d^{2 n} \zeta$. This means for smooth functions $f_{i} \in \mathcal{C}^{\infty}\left(\mathcal{O}_{\Lambda}\right)$ that $I_{N}\left[P_{N}\left(f_{1}\right) P_{N}\left(f_{2}\right)\right](o) \rightarrow f_{1}(o) f_{2}(o)$ as $N \rightarrow \infty$, and (using the homogeneity of $\mathcal{O}_{\Lambda}$ )

$$
I_{N}\left[P_{N}\left(f_{1}\right) P_{N}\left(f_{2}\right)\right] \underset{N \rightarrow \infty}{\longrightarrow} f_{1} f_{2} .
$$

If, instead of smooth functions, we have continuous functions $f_{i} \in \mathcal{C}\left(\mathcal{O}_{\Lambda}\right)$ then we can approximate these with smooth functions $\tilde{f}_{i}$. Because the maps $I_{N}$ and $P_{N}$ are completely positive, they are norm-contracting; this implies that the norm-difference

$$
\left\|I_{N}\left[P_{N}\left(f_{1}\right) P_{N}\left(f_{2}\right)\right]-I_{N}\left[P_{N}\left(\tilde{f}_{1}\right) P_{N}\left(\tilde{f}_{2}\right)\right]\right\|
$$

is bounded uniformly as $N \rightarrow \infty$ and goes to 0 as $\tilde{f}_{i} \rightarrow f_{i}$. This means that (5.16) is true for all continuous functions.

Using the fact that $P_{N}(1)=1$, this also shows that $I_{N}$ and $P_{N}$ are asymptotically inverse, in the sense that $I_{N} \circ P_{N}(f) \rightarrow f$ as $N \rightarrow \infty$. This property means that we can replace $P_{N}$ by a left inverse of $I_{N}$, and Eq. (5.16) will continue to hold. This shows that the direct limit converges (see App. B.1). Likewise, we can replace $I_{N}$ by a right inverse of $P_{N}$, and Eq. (5.16) will continue to hold. This shows that the inverse limit converges (see App. B.3).

5.4. Polynomials. In Appendix B.1, the limit $\lim _{\longrightarrow}\left\{\mathcal{A}_{*}, i_{*}\right\}$ is constructed by first constructing the limit Vec-lim $\left\{\mathcal{A}_{*}, i_{*}\right\}$ as a sequence of vector spaces and then completing to a $\mathrm{C}^{*}$-algebra. In the particular case of coadjoint orbits, Vec-lim$\left\{\mathcal{A}_{*}, i_{*}\right\}$ is itself interesting.

The algebra $\mathcal{C}\left(\mathcal{O}_{\Lambda}\right)$ is, as a $G$-representation, a closure of the direct sum of all its irreducible subrepresentations. On the other hand, each $\mathcal{A}_{N}$ is finite-dimensional and is therefore just a direct sum of irreducibles; any element of the limit Vec-lim $\left\{\mathcal{A}_{*}, i_{*}\right\}$ is in the image of some $\mathcal{A}_{N}$; therefore, Vec-lim $\left\{\mathcal{A}_{*}, i_{*}\right\}$ is the "algebraic" direct sum of irreducibles. Since $\mathcal{C}\left(\mathcal{O}_{\Lambda}\right)$ is a closure of this, Vec-lim $\left\{\mathcal{A}_{*}, i_{*}\right\}$ must be the direct sum of all the irreducible subrepresentations of $\mathcal{C}\left(\mathcal{O}_{\Lambda}\right)$.

The polynomial functions $\mathbb{C}\left[\mathcal{O}_{\Lambda}\right]$ on $\mathcal{O}_{\Lambda}$ are defined as the restrictions to $\mathcal{O}_{\Lambda}$ of polynomials on $\mathfrak{g}^{*}$. The space of polynomials of a given degree is a direct sum of irreducible representations. Any polynomial has finite degree; therefore $\mathbb{C}\left[\mathcal{O}_{\Lambda}\right]$ is a direct sum of irreducible representations. Since $\mathbb{C}\left[\mathcal{O}_{\Lambda}\right]$ is dense in $\mathcal{C}\left(\mathcal{O}_{\Lambda}\right)$, it must be the direct sum of the irreducible subrepresentations of $\mathcal{C}\left(\mathcal{O}_{\Lambda}\right)$.

This shows that Vec-lim $\left\{\mathcal{A}_{*}, i_{*}\right\}=\mathbb{C}\left[\mathcal{O}_{\Lambda}\right]$, and so the vector space direct limit is in this case an algebra. Whether this is true in any more general case remains to be seen. 


\section{Quantization of Vector Bundles over $\mathcal{O}_{\Lambda}$}

6.1. General quantized bundles. $\mathcal{A}_{N} \equiv \operatorname{End}(N \Lambda)$ is a full (a. k. a. simple) matrix algebra. The classification of the modules of a full matrix algebra is elementary. Any module is a tensor product of the fundamental module with some vector space. In this case the fundamental module is $(N \Lambda)$, and the vector space should be a $G$ representation. Any irreducible, equivariant module of $\mathcal{A}_{N}$ must be of the form

$$
V_{N}=(N \Lambda) \otimes(\nu),
$$

with the algebra only acting on the first factor. Any finitely generated, equivariant $\mathcal{A}_{N}$-module is a direct sum of such irreducibles. Because $\mathcal{A}_{N}$ is finite-dimensional, this $V_{N}$ is automatically projective.

The defining property of a finitely generated, projective module is that it is a (complemented) submodule of the algebra $\mathcal{A}_{N}$ tensored with some vector space. This submodule can be picked out by a projection (idempotent). In the $G$-equivariant case, "vector space" becomes "G-representation", and the projection must be $G$ invariant. In the case of this $V_{N}$, the representation we tensor with can be chosen to be irreducible; call it $(\mu)$. This means that we can identify $V_{N}$ with a submodule of $\mathcal{A}_{N} \otimes(\mu)$ in the form

$$
V_{N}=\left[\mathcal{A}_{N} \otimes(\mu)\right] \cdot Q_{N},
$$

where $Q_{N}=Q_{N}^{2}$. The factor $(N \Lambda)$ is treated as a space of column vectors, but the factor $\left(N \Lambda^{*}\right) \otimes(\mu)$ is treated as a space of row vectors, i. e., $Q_{N}$ multiplies them from the right. Acting from the left, $Q_{N}$ would multiply the corresponding (dual) space of column vectors $(N \Lambda) \otimes\left(\mu^{*}\right)$; therefore $Q_{N} \in \operatorname{End}\left[(N \Lambda) \otimes\left(\mu^{*}\right)\right]=\mathcal{A}_{N} \otimes \operatorname{End}\left(\mu^{*}\right)$. We can choose $\mu$ such that $Q_{N}$ is the unique invariant projection from $(N \Lambda) \otimes\left(\mu^{*}\right)$ to the irreducible subrepresentation $\left(\nu^{*}\right)$.

The injection $i_{N}: \mathcal{A}_{N} \hookrightarrow \mathcal{A}_{N+1}$ can be applied to the tensor product of $\mathcal{A}_{N}$ with a fixed algebra - in this case $\operatorname{End}\left(\mu^{*}\right)$. Let us apply this to $Q_{N}$ and call the result $Q_{N+1}$; by Eq. (5.6), this is

$$
Q_{N+1}:=\left[i_{N} \otimes \mathrm{id}\right]\left(Q_{N}\right)=\left(\Pi_{+} \otimes 1\right)\left(1 \otimes Q_{N}\right)\left(\Pi_{+}^{*} \otimes 1\right) .
$$

$Q_{N+1}$ is an endomorphism on $([N+1] \Lambda) \otimes\left(\mu^{*}\right)$ and is clearly self-adjoint. Let $\psi \in$ $([N+1] \Lambda) \otimes\left(\mu^{*}\right)$ be a normalized vector, and look at the product

$$
\left\langle\psi\left|Q_{N+1}\right| \psi\right\rangle=\left\langle\left(\Pi_{+}^{*} \otimes 1\right) \psi\left|\left(1 \otimes Q_{N}\right)\right|\left(\Pi_{+}^{*} \otimes 1\right) \psi\right\rangle .
$$

Note that $\Pi_{+}^{*} \otimes 1$ is just the natural isometric inclusion of $([N+1] \Lambda)$ into $(\Lambda) \otimes(N \Lambda)$. The product (6.4) is equal to 1 if and only if $\left(\Pi_{+}^{*} \otimes 1\right) \psi$ is in the image $(\Lambda) \otimes\left(\nu^{*}\right)$ of $Q_{N}$; but since $\psi \in([N+1] \Lambda) \otimes\left(\mu^{*}\right)$, this is equivalent to $\psi$ lying in the intersection $\left(\Lambda+\nu^{*}\right)$. Conversely, (6.4) is 0 if $\left(\Pi_{+}^{*} \otimes 1\right) \psi$ is orthogonal to $(\Lambda) \otimes\left(\nu^{*}\right)$, or equivalently, if $\psi$ is orthogonal to $\left(\Lambda+\nu^{*}\right)$. This shows that $Q_{N+1}$ is the projection with image $\left(\Lambda+\nu^{*}\right)$.

Note that $\Pi_{+}^{*} \Pi_{+}$is the self-adjoint idempotent acting on $(\Lambda) \otimes(N \Lambda)$, with image $([N+1] \Lambda)$. Using the same sort of reasoning as in the last paragraph, the image of 
$\left(1 \otimes Q_{N}\right)\left(\Pi_{+}^{*} \otimes 1\right)$ is in $([N+1] \Lambda) \otimes\left(\mu^{*}\right)$, so there is the identity

$$
\begin{aligned}
\left(1 \otimes Q_{N}\right)\left(\Pi_{+}^{*} \otimes 1\right) & =\left(\Pi_{+}^{*} \Pi_{+} \otimes 1\right)\left(1 \otimes Q_{N}\right)\left(\Pi_{+}^{*} \otimes 1\right) \\
& =\left(\Pi_{+}^{*} \otimes 1\right) Q_{N+1} .
\end{aligned}
$$

In words, moving $1 \otimes Q_{N}$ right past $\Pi_{+}^{*} \otimes 1$ transforms it into $Q_{N+1}$.

The new projection $Q_{N+1}$ gives an $\mathcal{A}_{N+1}$-module

$$
V_{N+1}=\left[\mathcal{A}_{N+1} \otimes\left(\mu^{*}\right)\right] \cdot Q_{N+1}=([N+1] \Lambda) \otimes\left(\nu+\Lambda^{*}\right) .
$$

Repeating this process gives a whole sequence of modules. Since the weight in the second factor is changed by $\Lambda^{*}$ with each step, it is simpler to write in terms of $\lambda=\nu-N \Lambda^{*}$. The sequence of modules is now

$$
V_{N}^{\lambda}:=(N \Lambda) \otimes\left(N \Lambda^{*}+\lambda\right)
$$

Each of these can be realized as a submodule of $\mathcal{A}_{N} \otimes(\mu)$ in the form

$$
V_{N}^{\lambda}=\left[\mathcal{A}_{N} \otimes(\mu)\right] \cdot Q_{N}^{\lambda}
$$

The projections are related by the recursion?

$$
Q_{N+1}^{\lambda}=\left[i_{N} \otimes \mathrm{id}\right]\left(Q_{N}^{\lambda}\right)
$$

Because the construction of the $p_{N}$ 's is so similar to that of the $i_{N}$ 's, the same reasoning shows that $\left[p_{N} \otimes \mathrm{id}\right]\left(Q_{N}\right)$ is a projection as well. In fact, the same sequence of projections given by (6.8a) also satisfies

$$
Q_{N-1}^{\lambda}=\left[p_{N} \otimes \mathrm{id}\right]\left(Q_{N}^{\lambda}\right)
$$

Now, we can put all these $Q_{N}^{\lambda}$ 's together to form $Q^{\lambda} \in \Gamma\left(\mathcal{A}_{\mathbb{N}}\right) \otimes \operatorname{End}\left(\mu^{*}\right)$. The constructions of $\mathbb{A}$ in Appendices $\mathbb{B} .1$ and $\mathbb{B} .3$ say essentially that $Q^{\lambda} \in \mathbb{A} \otimes \operatorname{End}\left(\mu^{*}\right)$ if and only if one of the relations (6.8) is true in a limiting sense as $N \rightarrow \infty$. Since Eq.'s (6.8) are true for finite $N$, we have more than we need to show that $Q^{\lambda} \in$ $\mathbb{A} \otimes \operatorname{End}\left(\mu^{*}\right)$.

By construction, this $Q^{\lambda}$ is obviously a projection. Using this, we define

$$
\mathbb{V}^{\lambda}:=[\mathbb{A} \otimes(\mu)] \cdot Q^{\lambda} .
$$

This is a well defined, finitely generated, projective module of $\mathbb{A}$, and the restriction to each $\mathcal{A}_{N}$ is $V_{N}^{\lambda}$. This shows that $\mathbb{V}^{\lambda}$ is a general quantization of some bundle $V^{\lambda}$ over $\mathcal{O}_{\Lambda}$. Although $(\mu)$ was used in this construction, $\lambda$ completely determines $\mathbb{V}^{\lambda}$ as an $\mathbb{A}$-module.

\footnotetext{
${ }^{8}$ Actually, this is not quite always true; see Sec. 6.4.
} 
6.2. Limit quantized bundles. We can use $i_{N}$ to map

$$
i_{N} \otimes \text { id }: \mathcal{A}_{N} \otimes(\mu) \hookrightarrow \mathcal{A}_{N+1} \otimes(\mu) .
$$

For some $\psi \in \mathcal{A}_{N} \otimes(\mu)$, look at what happens to the product $\psi Q_{N}^{\lambda}$; using (6.5),

$$
\begin{aligned}
{\left[i_{N} \otimes \mathrm{id}\right]\left(\psi Q_{N}^{\lambda}\right) } & =\Pi_{+}\left(1 \otimes\left[\psi Q_{N}^{\lambda}\right]\right)\left(\Pi_{+}^{*} \otimes 1\right) \\
& =\Pi_{+}(1 \otimes \psi)\left(1 \otimes Q_{N}^{\lambda}\right)\left(\Pi_{+}^{*} \otimes 1\right) \\
& =\Pi_{+}(1 \otimes \psi)\left(\Pi_{+}^{*} \otimes 1\right) \cdot Q_{N+1}^{\lambda} \\
& =\left[i_{N} \otimes \mathrm{id}\right](\psi) \cdot Q_{N+1}^{\lambda} .
\end{aligned}
$$

This implies that $i_{N} \otimes$ id maps the image $V_{N}^{\lambda}$ of $Q_{N}^{\lambda}$ to the image $V_{N+1}^{\lambda}$ of $Q_{N+1}^{\lambda}$, so we can restrict $i_{N} \otimes$ id to $V_{N}^{\lambda}$ and get a well defined injection $\iota_{N}: V_{N}^{\lambda} \hookrightarrow V_{N+1}^{\lambda}$. These injections make a directed system out of the $V_{N}^{\lambda}$ 's. Because of the simple relationship with the directed system $\left\{\mathcal{A}_{*}, i_{*}\right\}$, the system $\left\{V_{*}^{\lambda}, \iota_{*}\right\}$ inherits its convergence.

In an essentially identical way, we can construct $\pi_{N}: V_{N}^{\lambda} \rightarrow V_{N-1}^{\lambda}$ as the restriction of $p_{N} \otimes \mathrm{id}$. This gives a convergent inverse system $\left\{V_{*}^{\lambda}, \pi_{*}\right\}$.

In spite of the way that they were constructed, these $\iota_{N}$ 's and $\pi_{N}$ 's are independent of the $(\mu)$ that we use. We can use the unique natural projection

$$
\Pi_{\lambda+} \in \operatorname{Hom}_{G}\left[(\Lambda) \otimes\left(N \Lambda+\lambda^{*}\right),\left([N+1] \Lambda+\lambda^{*}\right)\right]
$$

to write (in a slight modification of (6.10a))

$$
\iota_{N}(\psi)=\Pi_{+}(1 \otimes \psi) \Pi_{\lambda+}^{*} \cdot
$$

In this form $\iota_{N}$ manifestly depends only on $\Lambda, N$, and $\lambda$. There is again a precisely analogous form for $\pi_{N}$.

It is easy to see that $\Pi_{0+}=\Pi_{+}$, so $\iota_{N}$ in (6.11) is a simple generalization of $i_{N}$ in (5.6). Analogous to the maps $I_{N}$ and $P_{N}$ for the algebras, there are maps $I_{N}^{V^{\lambda}}: V_{N}^{\lambda} \hookrightarrow V_{\infty}^{\lambda} \equiv \Gamma\left(V^{\lambda}\right)$ and $P_{N}^{V^{\lambda}}: V_{\infty}^{\lambda} \rightarrow V_{N}^{\lambda}$. These are easily constructed as restrictions of $I_{N} \otimes$ id and $P_{N} \otimes$ id.

These limit quantizations both produce the same $\mathbb{V}^{\lambda}$ as was constructed using $Q^{\lambda}$ in the previous section. These are, therefore, all quantizations of the same bundle $V^{\lambda}$.

\subsection{Identification with bundles.}

Notation. Since the Lie algebras $\mathfrak{g}$ and $\mathfrak{h}$ share the same Cartan subalgebra, their weights are naturally identified (App. D.3). Denote the $H$-representation with highest weight $\lambda$ by $[\lambda]$. Note that $\Psi^{\lambda} \in[\lambda] \subset(\lambda)$. Beware that $[\lambda]^{*}$ and $\left[\lambda^{*}\right]$ are not generally the same.

I have established that the irreducible equivariant bundles are given by dominant weights of $H$, and irreducible equivariant quantized bundles are given by weights of $G$. So, what is the correspondence?

Using the quotient homomorphism $\mathcal{P}: \mathbb{A} \rightarrow \mathcal{C}\left(\mathcal{O}_{\Lambda}\right)$, define the limit projection

$$
Q_{\infty}^{\lambda}:=[\mathcal{P} \otimes \mathrm{id}]\left(Q^{\lambda}\right) \in \mathcal{C}\left(\mathcal{O}_{\Lambda}\right) \otimes \operatorname{End}\left(\mu^{*}\right) ;
$$


this is naturally thought of as a projection-valued function on $\mathcal{O}_{\Lambda}$. The bundle $V^{\lambda}$ can be realized as the subbundle of $\mathcal{O}_{\Lambda} \times(\mu)$ determined by $Q_{\infty}^{\lambda}$. At each point $x \in \mathcal{O}_{\Lambda}$, the fiber of $V^{\lambda}$ is $V_{x}^{\lambda}=(\mu) \cdot Q_{\infty}^{\lambda}(x) \subset(\mu)$.

The injection $I_{N}$ is heuristically the limit of applying $i_{N}$, then $i_{N+1}$, then $i_{N+2}$, and so on. The recursion relation (6.8a) thus implies that $\left[I_{N} \otimes \mathrm{id}\right]\left(Q_{N}^{\lambda}\right)=Q_{\infty}^{\lambda}$.

As explained in Section 4.2, the equivariant bundle $V^{\lambda}$ is completely determined by its fiber at $o \in \mathcal{O}_{\Lambda}$. This fiber is given by $Q_{\infty}^{\lambda}$ as $V_{o}^{\lambda}=(\mu) \cdot Q_{\infty}^{\lambda}(o)$. It is more convenient to first determine the dual $\left(V_{o}^{\lambda}\right)^{*}=Q_{\infty}^{\lambda}(o) \cdot\left(\mu^{*}\right)$.

The $H$-representation $\left(V_{o}^{\lambda}\right)^{*}$ is the image of $Q_{\infty}^{\lambda}(o)$. This is actually an irreducible representation, so it is determined by its highest weight. Let $\psi \in\left(\mu^{*}\right)$ be a normalized vector of a given weight. If (and only if) $\psi \in\left(V_{o}^{\lambda}\right)^{*}$ then $\left\langle\psi\left|Q_{\infty}^{\lambda}(o)\right| \psi\right\rangle=1$. So, evaluate this expression; it is (using (5.5))

$$
\begin{aligned}
\left\langle\psi\left|Q_{\infty}^{\lambda}(o)\right| \psi\right\rangle & =\left\langle\psi\left|\left[\left(I_{N} \otimes \mathrm{id}\right)\left(Q_{N}^{\lambda}\right)\right](o)\right| \psi\right\rangle \\
& =\left\langle\Psi^{N \Lambda} \otimes \psi\left|Q_{N}^{\lambda}\right| \Psi^{N \Lambda} \otimes \psi\right\rangle .
\end{aligned}
$$

This is 1 if and only if $\Psi^{N \Lambda} \otimes \psi \in\left(N \Lambda+\lambda^{*}\right)$. Since $N \Lambda+\lambda^{*}$ is the highest weight of $\left(N \Lambda+\lambda^{*}\right)$, the highest weight that $\psi$ can have under this condition is $\lambda^{*}$. This means that $\left(V_{o}^{\lambda}\right)^{*}=\left[\lambda^{*}\right]$; therefore $V_{o}^{\lambda}=\left[\lambda^{*}\right]^{*}$. Finally, this gives

$$
V^{\lambda}=\left[\lambda^{*}\right]^{*} \times_{H} G \text {. }
$$

6.4. The allowed weights. The recursion relation (6.8a) is actually not true for quite all values of $\lambda$ and $N$.

If a weight $\nu$ is not dominant, then there really is no representation $(\nu)$. It is, however, convenient to define $(\nu):=0$ in that case. The condition that $\mathbb{V}^{\lambda} \neq 0$ is that $N \Lambda^{*}+\lambda$ is dominant for some $N$.

If $\lambda$ satisfies this condition but is not itself dominant, then for low $N$ values $V_{N}^{\lambda}=0$, but for sufficiently large $N$ values $V_{N}^{\lambda} \neq 0$. In this case there is some $N$ such that $V_{N}^{\lambda}=0 \neq V_{N+1}^{\lambda}$. This means that $Q_{N}^{\lambda}=0 \neq Q_{N+1}^{\lambda}$, so obviously $\left[i_{N} \otimes \mathrm{id}\right]\left(Q_{N}\right)=0 \neq$ $Q_{N+1}^{\lambda}$ and (6.8a) fails. However, this is the only time that (6.8a) is not true, so there is no real trouble from this. Equation (6.8b), on the other hand, is always true.

The condition that $V^{\lambda}$, as given by (6.12), is a nonzero bundle is that $\lambda^{*}$ is dominant as an $H$-weight. This is actually exactly equivalent to the condition just described for $\mathbb{V}^{\lambda} \neq 0$. This means that any finitely generated, locally trivial, equivariant vector bundle can be equivariantly quantized.

\section{Further Remarks on Bundles}

7.1. Uniqueness. Equivariant bundles and modules are classified by equivariant $K$-theory. The equivariant vector bundles over $\mathcal{O}_{\Lambda}$ all have equivalence classes in $K_{G}^{0}\left(\mathcal{O}_{\Lambda}\right)$. As has been mentioned (Sec. 4.2), these bundles are classified by representations of $H$. From this it is easy to see that an equivariant bundle is uniquely specified by its $K$-class. Similarly, an equivariant module of $\mathbb{A}$ is uniquely specified by its $K$-class in $K_{0}^{G}(\mathbb{A})$. The equivariant general quantizations of vector bundles are equivariant modules of $\mathbb{A}$, and are thus classified by $K_{0}^{G}(\mathbb{A})$. Since $\mathcal{C}\left(\mathcal{O}_{\Lambda}\right)=\mathbb{A} / \mathbb{A}_{0}$, 
there is a corresponding six-term periodic exact sequence in $K$-theory. Part of this sequence reads

$$
K_{0}^{G}\left(\mathbb{A}_{0}\right) \rightarrow K_{0}^{G}(\mathbb{A}) \rightarrow K_{G}^{0}\left(\mathcal{O}_{\Lambda}\right) \rightarrow K_{1}^{G}\left(\mathbb{A}_{0}\right)
$$

The ideal $\mathbb{A}_{0}=\Gamma_{0}\left(\mathcal{A}_{\mathbb{N}}\right)$ is the $\mathrm{C}^{*}$-direct sum of the algebras $\mathcal{A}_{N}$; therefore $K_{*}^{G}\left(\mathbb{A}_{0}\right)=$ $\bigoplus_{N=1}^{\infty} K_{*}^{G}\left(\mathcal{A}_{N}\right)$. Because $\mathcal{A}_{N}$ is the matrix algebra on a simple representation of $G$, its equivariant $K$-theory is very simple. In degree $0, K_{0}^{G}\left(\mathcal{A}_{N}\right)=\mathcal{R}(G)$ the unitary representation ring of $G$. In degree $1, K_{1}^{G}\left(\mathcal{A}_{N}\right)=0$. This simplifies the exact sequence (7.1). Now it reads

$$
\mathcal{R}(G)^{\oplus \infty} \rightarrow K_{0}^{G}(\mathbb{A}) \rightarrow K_{G}^{0}\left(\mathcal{O}_{\Lambda}\right) \rightarrow 0 .
$$

Firstly, this shows that — at the level of $K$-theory — any equivariant bundle has an equivariant quantization, since it has a preimage in $K_{0}^{G}(\mathbb{A})$. This corroborates the conclusion of Section 6.3. Secondly, this describes the variety of possible quantizations of a given bundle. If two equivariant $\mathbb{A}$-modules quantize the same bundle, then the difference of their $K$-classes is in the image of $\mathcal{R}(G)^{\oplus \infty}$, but that is an algebraic direct sum; it consists of sequences with only finitely many nonzero terms, and each term concerns a single $N$. This means that if both $V_{\mathbb{N}}$ and $V_{\mathbb{N}}^{\prime}$ are quantizations of $V$, then for all $N$ sufficiently large, $V_{N} \cong V_{N}^{\prime}$.

Given this conclusion, the choice of $V_{N}$ 's in Eq. (6.6) must be the unique one given by a simple formula.

7.2. Geometric Quantization. For each $N$, the fundamental module $(N \Lambda)$ of $\mathcal{A}_{N}$ is of course a module. It is tempting to ask if these together form the quantization of some bundle, but they do not. The $\mathbb{A}$-module formed by assembling these is not projective.

It is reasonable to instead ask - separately for each $N$ - what bundle's equivariant quantization (by the construction of Sec. 6) has $V_{N}=(N \Lambda)$ ? This is easily answered;

$$
(N \Lambda)=(N \Lambda) \otimes(0)=(N \Lambda) \otimes\left(N \Lambda^{*}-N \Lambda^{*}\right)=V_{N}^{-N \Lambda^{*}} .
$$

Using the identity that $[N \Lambda]^{*}=[-N \Lambda]$ (see App. D.3), the corresponding bundle is $V^{-N \Lambda^{*}}=[N \Lambda] \times{ }_{H} G$.

The $H$-representation $[N \Lambda]$ is one-dimensional; this bundle is therefore of rank 1 - i. e., it is a line bundle. In geometric quantization of $\mathcal{O}_{\Lambda}$, the fundamental module $(N \Lambda)$ of $\mathcal{A}_{N}$ is constructed as the space of holomorphic sections of this very line bundle.

7.3. Bimodules. For a commutative algebra, any module can automatically be considered a bimodule; simply define right multiplication to be equal to left multiplication. However, it is not generally the case that when a vector bundle is quantized, the corresponding module continues to be a bimodule. The right side (the row-vector factor) of $V_{N}^{\lambda}$ is $\left(N \Lambda^{*}+\lambda\right)$ and does not in general admit any equivariant right multiplication by $\mathcal{A}_{N} \equiv \operatorname{End}(N \Lambda)$.

If $V_{N}$ is an $\mathcal{A}_{N}$-bimodule, then it must contain a factor of $(N \Lambda)$ to accommodate the left multiplication, and a separate factor of $\left(N \Lambda^{*}\right)$ to accommodate the right 
multiplication. It must therefore be the tensor product of $\mathcal{A}_{N}$ itself by some representation. The corresponding classical bundle is then the trivial bundle with fiber equal to that representation. This is an unpleasantly restrictive class.

A slightly broader class of bundles results if we allow the quantum modules to be multiplied from the left and right by different $\mathcal{A}_{N}$ 's. This is enough to make $\mathbb{V}$ an $\mathbb{A}$-bimodule, and is also contrary to the philosophy of each $N$ being a separate step along the way to the classical limit. The irreducibles of this class of modules are of the form

$$
V_{N}=(N \Lambda) \otimes([N+m] \Lambda) \otimes(\lambda)=V_{N}^{m \Lambda^{*}} \otimes(\lambda) .
$$

The corresponding classical bundles are a slightly more interesting class than trivial bundles, but still quite restrictive. This can be extended a little further in some cases by using a larger parameter set $\mathcal{I}$. It remains to be seen whether this class of modules is useful.

\section{The CASE OF The 2-SPhere}

The group $\mathrm{SU}(2)$ is the most elementary compact, simple Lie group, so the simplest example of what has been described here is for $G=\mathrm{SU}(2)$. There is only one distinct coadjoint orbit for $\mathrm{SU}(2)$; it is the 2-sphere. As a coset space $S^{2}=\mathrm{SU}(2) / \mathrm{U}(1)$.

The positive Weyl chamber of $\mathrm{SU}(2)$ is $\mathcal{C}_{+}=\mathbb{R}_{+}$. Thought of as the parameter space for $S^{2}$ 's, this is the set of radii. In deference to standard physics notation, I will identify the dominant weights with positive half-integers. The irreducible representations are thus $(0),\left(\frac{1}{2}\right),(1)$, et cetera. The most appropriate choice for $\Lambda$ is $\frac{1}{2}$.

The Lie algebra $\mathfrak{s u}(2)$ is generated by $J_{1}, J_{2}$, and $J_{3}$, with the commutation relations $\left[J_{i}, J_{j}\right]_{-}=i \epsilon_{i j}^{k} J_{k}$; that is, $\left[J_{1}, J_{2}\right]_{-}=i J_{3}$, et cetera.

The standard choice for the Cartan subalgebra $\mathfrak{C}$ is the one-dimensional span of $J_{3}$. The weights are just the eigenvalues of $J_{3}$. In the representation $\left(\frac{N}{2}\right)$ the highest weight vector satisfies $J_{3} \Psi^{N / 2}=\frac{N}{2} \Psi^{N / 2}$.

There is a single (quadratic) Casimir operator $\mathcal{C}_{1}(J)=J^{2} \equiv J_{1}^{2}+J_{2}^{2}+J_{3}^{2}$. Its eigenvalue on the representation $\left(\frac{N}{2}\right)$ is $\frac{N}{2}\left[\frac{N}{2}+1\right]$.

There is a single Serre relation for $\operatorname{End}\left(\frac{N}{2}\right)$. In terms of the element $J_{+}:=\frac{1}{2}\left(J_{1}+\right.$ $\left.i J_{2}\right)$, the relation is that $J_{+}^{N+1}=0$. Although this is expressed in a noninvariant way, this condition really is invariant; it could equivalently be expressed in terms of many other possible combinations of $J$ 's.

The logic of the Serre relation is that the representation $\left(\frac{N}{2}\right)$ is $N+1$ dimensional. It can be decomposed into one-dimensional weight subspaces ( $J_{3}$ eigenspaces). The operator $J_{+}$shifts these weight subspaces; it maps the subspace with weight $m$ to the subspace with weight $m+1$ (the next higher possible weight). $J_{+}$can be applied to some $J_{3}$-eigenvector no more than $N$ times before there are no more eigenvalues available, and the result must be 0 . Therefore $J_{+}^{N+1}$ applied to anything in $\left(\frac{N}{2}\right)$ must give 0 . 
We can construct a general quantization by the method of Section 5.1. The generators $x_{i}:=\mathcal{P}\left(N^{-1} J_{i}\right)$ of the resulting $\mathcal{A}_{\infty}$ satisfy the relations of commutativity and $x_{1}^{2}+x_{2}^{2}+x_{3}^{2}=\frac{1}{4}$. Obviously this shows $\mathcal{A}_{\infty}$ to be the continuous functions on the sphere of radius $\frac{1}{2}$ in $\mathfrak{s u}(2)^{*} \cong \mathbb{R}^{3}$.

All SU(2)-representations are self-dual. Because of this, the constructions of $i_{N}$ and $p_{N}$ are even closer than in the general case in Section 5.2. Decompose the tensor product $\left(\frac{1}{2}\right) \otimes\left(\frac{N}{2}\right)=\left(\frac{N+1}{2}\right) \oplus\left(\frac{N-1}{2}\right)$. There is a representation of $\mathcal{A}_{N}$ on this that acts trivially on the $\left(\frac{1}{2}\right)$ factor; for an element $a \in \mathcal{A}_{N}$, the $\left(\frac{N+1}{2}\right)$ corner of this representation matrix is $i_{N}(a) \in \mathcal{A}_{N+1}$; the $\left(\frac{N-1}{2}\right)$ corner is $p_{N}(a) \in \mathcal{A}_{N-1}$.

In this case it is possible to construct a simple and (partly) explicit formula for the "product" kernel $K_{N}$. The key is to use the identification $S^{2}=\mathbb{C P}^{1}=\mathbb{P}\left(\frac{1}{2}\right)$. The geodesic distances on $S^{2}$ are given by the Fubini-Study metric; for two points $[\psi],[\varphi] \in \mathbb{P}\left(\frac{1}{2}\right)$ the distance $d_{S^{2}}([\psi],[\varphi])$ is determined by

$$
\cos ^{2}\left[d_{S^{2}}([\psi],[\varphi])\right]:=\frac{|\langle\psi \mid \varphi\rangle|^{2}}{\langle\psi \mid \psi\rangle\langle\varphi \mid \varphi\rangle} .
$$

Recall that this is the sphere of radius $\frac{1}{2}$, so $0 \leq d_{S^{2}}(x, y) \leq \frac{\pi}{2}$. Comparing this with the formulas (5.12) or (E.2) for $K_{1}$ gives that

$$
\left|K_{1}(x, y, z)\right|=4 \cdot \cos \left[d_{S^{2}}(x, y)\right] \cos \left[d_{S^{2}}(y, z)\right] \cos \left[d_{S^{2}}(z, x)\right] .
$$

Noting (5.14), this gives for arbitrary $N$ that

$$
\left|K_{N}(x, y, z)\right|=(N+1)^{2} \cos ^{N}\left[d_{S^{2}}(x, y)\right] \cos ^{N}\left[d_{S^{2}}(y, z)\right] \cos ^{N}\left[d_{S^{2}}(z, x)\right] .
$$

What remains to be determined is the phase. This has no simple formula, but is easily understood geometrically: $\arg K_{N}(x, y, z)$ is $2 N$ times the area of the geodesic triangle on $S^{2}$ with vertices $x, y$, and $z$. To see this, show that this is true for infinitesimal triangles and that this quantity is additive when a triangle is decomposed into smaller triangles. Clearly $K_{N}$ does indeed become sharply peaked as $N \rightarrow \infty$.

Since the isotropy group of $S^{2}$ is $H=\mathrm{U}(1)$, the classification of equivariant vector bundles over $S^{2}$ is extremely simple. The irreducible bundles are classified by irreducible representations of $\mathrm{U}(1)$, which are in turn indexed by half integers. Denote these representations by $[m]$ for any $m \in \frac{1}{2} \mathbb{Z}$. Since these representations are all one-dimensional, the irreducible bundles are all rank-one.

Under the restriction $\mathrm{SU}(2) \hookleftarrow \mathrm{U}(1)$, an irreducible representation of $\mathrm{SU}(2)$ decomposes into a direct sum of irreducible $\mathrm{U}(1)$-representations. This is simply

$$
(j) \rightarrow[-j] \oplus[-j+1] \oplus \cdots \oplus[j]
$$

for any $j \in \frac{1}{2} \mathbb{Z}$.

Let $W^{m}:=[m] \times_{\mathrm{U}(1)} \mathrm{SU}(2)$ be the equivariant vector bundle over $S^{2}$ with fiber $W_{o}^{m}=[m]$. The space of continuous sections $\Gamma\left(W^{m}\right)$ is a completion of the space of polynomial sections $\Gamma_{\text {poly }}\left(W^{m}\right)$. As an $\mathrm{SU}(2)$-representation, $\Gamma_{\text {poly }}\left(W^{m}\right)$ is a direct sum of irreducibles and is easily computed. The representation $(j)$ occurs precisely 
once in $\Gamma_{\text {poly }}\left(W^{m}\right)$ if and only if $[m]$ occurs in the decomposition of $(j)$; in other words, when $j \equiv m \bmod 1$ and $j \geq|m|$. So,

$$
\Gamma_{\text {poly }}\left(W^{m}\right)=(|m|) \oplus(|m|+1) \oplus(|m|+2) \oplus \cdots .
$$

So, what is the quantization of $W^{m}$ ? We need to know for which $j$ does $W^{m}=V^{j}$. Equation (6.12) shows that $[m]=\left[j^{*}\right]^{*}$. Since all $\mathrm{SU}(2)$-representations are self-dual, $j^{*}=j$. All irreducible U(1)-representations are one-dimensional, so (see App. D.3) $[j]^{*}=[-j]$. This means that $j=-m$, and the quantization of $W^{m}$ is $\mathbb{V}^{-m}$.

As an $\mathrm{SU}(2)$ representation

$$
V_{N}^{-m}=\left(\frac{N}{2}\right) \otimes\left(\frac{N}{2}-m\right)=(|m|) \oplus(|m|+1) \oplus \cdots \oplus(N-m) .
$$

Clearly, modulo completion, $V_{N}^{-m}$ in the limit $N \rightarrow \infty$ becomes the same $\mathrm{SU}(2)$ representation as $\Gamma\left(W^{m}\right)$. This applies in particular when $\lambda=0$ so $V_{N}^{0}=\mathcal{A}_{N}$ and $\Gamma\left(W^{0}\right)=\mathcal{C}\left(S^{2}\right)$. With a little more work, this consistency check can be carried out for all the complex projective spaces $\mathbb{C P}^{n}$.

In a way, it may seem odd to be using $\mathrm{SU}(2)$ as the symmetry group for $S^{2}$. The group of distinct orientation preserving isometries of $S^{2}$ is $\mathrm{SO}(3)$; the group $\mathrm{SU}(2)$ is its simply-connected, double cover. If we had used the smaller group, we would have artificially excluded all the $\mathcal{A}_{N}$ 's with $N$ odd. Although $\mathrm{SO}(3)$ acts on all the algebras $\mathcal{A}_{N}$, we need the $\mathrm{SU}(2)$-representation $(N \Lambda)$ in order to construct $\mathcal{A}_{N}$. Another reason is that many of the vector bundles on $S^{2}$ are $\mathrm{SU}(2)$-equivariant, but not $\mathrm{SO}(3)$-equivariant.

It is generally the case that the simply connected $G$ is not the minimal symmetry group of a coadjoint orbit. Indeed, the minimal symmetry group of $\mathcal{O}_{\Lambda}$ is the group $G^{\prime}=G / Z(G)$ (the "adjoint group") which maximizes the fundamental group $\pi_{1}\left(G^{\prime}\right)$. Nevertheless, the simply connected $G$ is the easiest to deal with, and most fruitful, choice.

\section{FinAl REMARKS}

One motivation for considering the limit quantization approach for bundles comes from physics. If this sort of quantization is used as a regularization technique, then it would be desirable to do a "renormalization group" analysis. This involves going from one level of regularization to a coarser one with fewer degrees of freedom. In order to do this we need a sort of coarse-graining map that associates a given field configuration with a coarser field configuration, ignoring some of the degrees of freedom of the original.

In $n$-dimensional lattice regularization, the space is approximated by a lattice. The coarse-graining is accomplished by grouping the lattice points into groups of $2^{n}$ and averaging the field values at those $2^{n}$ points. This field value is then given to a single point of the new, coarsened lattice which has $2^{-n}$ times as many points. The degrees of freedom are thus reduced (drastically) by a factor of $2^{n}$.

Classically, field configurations are sections of vector bundles. If quantization is used as a regularization technique, the field configurations are the vectors in the 
quantum modules $V_{N}$. Coarse-graining means going from $N$ to $N-1$. The coarsegraining map is $\pi_{N}$. The degrees of freedom vary as only a polynomial function of $N$, so $\operatorname{dim} V_{N} / \operatorname{dim} V_{N-1} \approx 1$ for large $N$. This is far gentler than lattice regularization. I hope to discuss this, and related matters in a future paper.

Another reason for using constructions in terms of limits, as I have here, is simply that it is the most convenient approach when dealing with coadjoint orbits. When dealing with the quantization of a more general symplectic manifold, objects such as the Hilbert space $\mathcal{H}_{N}$ are constructed as spaces of sections over the manifold; everything is constructed from the manifold. In the case of coadjoint orbits, however, $\mathcal{H}_{N}$ is constructed directly as a $G$-representation. We can actually deal more explicitly with the algebra $\mathcal{A}_{N}$ than with the algebra $\mathcal{C}\left(\mathcal{O}_{\Lambda}\right)$. For this reason, it is more convenient to construct the classical structures from the quantum structures, rather than vice versa.

The construction of the maps $I_{N}$ and $P_{N}$ in Section 5.2 is standard [1, 12, 18]. In the terminology of Berezin [1], $P_{N}(a)$ is the contravariant symbol of $a$, and an element of the preimage $I_{N}^{-1}(a)$ is a covariant symbol of $a$.

The idea of directed limit quantization here is based on a construction by Grosse, Klimčík, and Prešnajder in [9]. In that case the quantization of the $S^{2}$ was being discussed. Their choice of $i_{N}$ is different and is based on the criterion of preserving the $L^{2}$-norm from one algebra to the next. My choice as based on the criterion of compatibility with the standard $I_{N}$ 's. It can easily be checked that $I_{N}$ never preserves the $L^{2}$ norms, and therefore my choice of $i_{N}$ 's never satisfies their criterion.

In [10], Grosse, Klimčík, and Prešnajder constructed quantized vector bundles for the special case of $S^{2}$. Their result is the same as mine for that case (see Sec. 8).

To reiterate, the main conclusion of this paper is that when the coadjoint orbit $\mathcal{O}_{\Lambda}=G / H$ through $\Lambda$ is quantized to give a sequence of matrix algebras

$$
\mathcal{A}_{N}=\operatorname{End}(N \Lambda)
$$

the equivariant vector bundle

$$
V^{\lambda}=\left[\lambda^{*}\right]^{*} \times_{H} G
$$

quantizes to a corresponding sequence of $\mathcal{A}_{N}$-modules

$$
V_{N}^{\lambda}=(N \Lambda) \otimes\left(N \Lambda^{*}+\lambda\right)
$$

In 114 I will continue by describing analogous results in the more general case of compact Kähler manifolds.

\section{Appendix A. SECtions}

Before discussing the construction of limits, it is worthwhile to clarify the notations for different spaces of sections of the bundles of algebras and modules. Given a noncompact base space, there are several useful types of continuous sections of a 
vector bundle, all of which are equivalent for a compact base space. For the base space $\mathbb{N}$, sections are the same thing as sequences. For legibility, I will sometimes write sections as sequences in that case.

The space of all continuous sections of a vector bundle $E$ is denoted $\Gamma(E)$. If $E$ is a bundle of algebras, then $\Gamma(E)$ is an algebra. However, for a bundle $\mathcal{A}_{\mathcal{I}}$ of $\mathrm{C}^{*}$-algebras $\Gamma\left(\mathcal{A}_{\mathcal{I}}\right)$ is not a $\mathrm{C}^{*}$-algebra since the sup-norm diverges. For a discrete base space, this is the algebraic direct product.

The space of continuous sections with compact support is denoted $\Gamma_{\mathrm{c}}(E)$. For a bundle of algebras, this is an ideal inside $\Gamma(E)$. For the $\mathrm{C}^{*}$-bundle $\mathcal{A}_{\mathcal{I}}$ this space $\Gamma_{\mathrm{c}}\left(\mathcal{A}_{\mathcal{I}}\right)$ has a $\mathrm{C}^{*}$-norm, but is not complete and therefore not $\mathrm{C}^{*}$. For a discrete base space, this is the algebraic direct sum.

If the fibers of $E$ are normed (as $\mathrm{C}^{*}$-algebras are), then two more types of section can be defined. $\Gamma_{\mathrm{b}}(E)$ is the space of sections of bounded norm. For the $\mathrm{C}^{*}$-bundle $\mathcal{A}_{\mathcal{I}}, \Gamma_{\mathrm{b}}\left(\mathcal{A}_{\mathcal{I}}\right)$ is a $\mathrm{C}^{*}$-algebra; the norm of a section is the supremum of the norms at all points of $\mathcal{I}$. For $\mathrm{C}^{*}$-algebras over a discrete base space this is the $\mathrm{C}^{*}$-direct sum.

$\Gamma_{0}(E)$ is the space of sections such that the norms converge to 0 approaching $\infty$. To be precise, any arbitrarily low bound on the norms is satisfied on the complement of some compact set. This is the norm closure of $\Gamma_{\mathrm{c}}(E)$. For the $\mathrm{C}^{*}$-bundle $\mathcal{A}_{\mathcal{I}}, \Gamma_{0}\left(\mathcal{A}_{\mathcal{I}}\right)$ is a closed ideal in $\Gamma_{\mathrm{b}}\left(\mathcal{A}_{\mathcal{I}}\right)$. For $\mathrm{C}^{*}$-algebras over a discrete base space, this is the $\mathrm{C}^{*}$-direct product.

These spaces of sections are related by $\Gamma_{\mathrm{c}} \subset \Gamma_{0} \subset \Gamma_{\mathrm{b}} \subset \Gamma$.

The appropriate notion of a bundle of $\mathrm{C}^{*}$-algebras is that of a continuous field of $\mathrm{C}^{*}$-algebras. This is discussed extensively in [6].

\section{APpendix B. Limits}

B.1. Direct limit of algebras. Since we are assuming the index set to be $\mathbb{N}$, sections of $\mathcal{A}_{\mathbb{N}}$ can also be thought of as sequences.

In the category of vector spaces, the limit of a directed system of algebras is

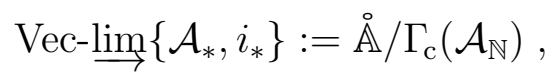

where

$$
\AA:=\left\{a \in \Gamma\left(\mathcal{A}_{\mathbb{N}}\right) \mid \exists M \forall N \geq M: a_{N+1}=i_{N}\left(a_{N}\right)\right\} .
$$

The injections $i_{N}$ are meant to identify $a_{N}$ to $i_{N}\left(a_{N}\right)$; (B.1) therefore gives the set of sequences which for sufficiently large $N$ become constant, modulo the sequences which for sufficiently large $N$ are 0 . Thinking of $\mathcal{A}_{N} \subset \mathcal{A}_{N+1}$, the limit is heuristically the union $\bigcup_{N \in \mathbb{N}} \mathcal{A}_{N}$ of this nested sequence.

Usually, one works in the category of $\mathrm{C}^{*}$-algebras in which the morphisms are *-homomorphisms. If the $i_{N}$ 's are assumed to be $*$-homomorphisms, then the $\mathrm{C}^{*}-$ algebraic limit (see [7]) of finite-dimensional algebras will be (by definition) an AFalgebra. This is far too restrictive a class of algebras in this context; a commutative AF-algebra is isomorphic to the continuous functions on a totally disconnected, zerodimensional space (see [21]). In order to avoid this restriction, we must allow the $i_{N}$ 's 
to be some more general type of morphism. Firstly, these must be linear, and I will assume (perhaps unnecessarily) that they are unital (i. e., $i_{N}(1)=1$ ).

Several convergence conditions on the $i_{N}$ 's will also be needed. The first condition is that the $i_{N}$ 's be norm-contracting maps; this means $\forall a \in \mathcal{A}_{N},\left\|i_{N}(a)\right\| \leq\|a\|$. There is a fairly nice class of norm-contracting maps for $\mathrm{C}^{*}$-algebras; these are the completely positive maps (see [16]). All of the $i_{N}$ 's and $p_{N}$ 's constructed in this paper are completely positive; however, I am not relying on that property in general. The norm-contracting condition ensures $\AA \subset \Gamma_{\mathrm{b}}\left(\mathcal{A}_{\mathbb{N}}\right)$.

Since each $\mathcal{A}_{N}$ is a $\mathrm{C}^{*}$-algebra, each has a $\mathrm{C}^{*}$-norm. The natural norm on the limit is the limit of these; that is, for any equivalence class $[a] \in \operatorname{Vec}-\underline{\lim }\left\{\mathcal{A}_{*}, i_{*}\right\}$ define

$$
\|[a]\|:=\lim _{N \rightarrow \infty}\left\|a_{N}\right\| .
$$

The norm-contracting condition guarantees that this is well defined, since it is a limit of a sequence that is (for sufficiently large $N$ ) strictly nonincreasing and bounded from below (by 0).

To ensure that this is truly a norm requires a second condition - that it be nondegenerate. That is, $a \neq 0 \Longrightarrow\|a\| \neq 0$. This is equivalent to the condition that $\AA \cap \mathbb{A}_{0}=\Gamma_{\mathrm{c}}\left(\mathbb{A}_{\mathbb{N}}\right)$, where $\mathbb{A}_{0}=\Gamma_{0}\left(\mathcal{A}_{\mathbb{N}}\right)$.

This means that $\Gamma_{\mathrm{c}}\left(\mathcal{A}_{\mathbb{N}}\right)$ can be replaced by $\mathbb{A}_{0}$ in $(\mathbb{B . 1})$, and $\AA$ is naturally embedded in the $\mathrm{C}^{*}$-algebra $\Gamma_{\mathrm{b}}\left(\mathcal{A}_{\mathbb{N}}\right) / \mathbb{A}_{0}$. The norm (B.3) agrees with the natural norm on this quotient. Now define $\mathcal{A}_{\infty}=\underline{\lim }\left\{\mathcal{A}_{*}, i_{*}\right\}$ as the closure of $\AA / \mathbb{A}_{0}$ in $\Gamma_{\mathrm{b}}\left(\mathcal{A}_{\mathbb{N}}\right) / \mathbb{A}_{0}$, or equivalently as the abstract norm completion of $\AA / \mathbb{A}_{0}$.

Also define $\mathbb{A} \subset \Gamma_{\mathrm{b}}\left(\mathcal{A}_{\mathbb{N}}\right)$ as the norm closure of $\AA \subset \Gamma_{\mathrm{b}}\left(\mathcal{A}_{\mathbb{N}}\right)$. Another construction of $\mathcal{A}_{\infty}$ is $\mathcal{A}_{\infty}=\mathbb{A} / \mathbb{A}_{0}$; this shows that if we view sections in $\Gamma_{\mathrm{b}}\left(\mathcal{A}_{\mathbb{N}}\right)$ as sequences, then $\mathbb{A}$ is the subspace of sequences which converge into $\mathcal{A}_{\infty}$.

It is not a priori true that $\mathcal{A}_{\infty}$ is an algebra; this requires a third (and final) condition. Require that $\mathcal{A}_{\infty}$ be algebraically closed in $\Gamma_{\mathrm{b}}\left(\mathcal{A}_{\mathbb{N}}\right) / \mathbb{A}_{0}$. This is equivalent to requiring that $\mathbb{A} \subset \Gamma_{\mathrm{b}}\left(\mathcal{A}_{\infty}\right)$ be algebraically closed.

Assuming these conditions, both $\mathcal{A}_{\infty}$ and $\mathbb{A}$ are norm closed subalgebras of $\mathrm{C}^{*}$ algebras; they are therefore $\mathrm{C}^{*}$-algebras themselves.

For each $N$, there is a canonical injection

$$
I_{N}: \mathcal{A}_{N} \hookrightarrow \operatorname{Vec}-\lim _{\longrightarrow}\left\{\mathcal{A}_{*}, i_{*}\right\} \subset \mathcal{A}_{\infty}
$$

which takes $a \mapsto\left[\left(0, \ldots, 0, a, i_{N}(a), i_{N+1} \circ i_{N}(a), \ldots\right)\right]$. Heuristically, $I_{N}=\ldots \circ i_{N+1} \circ$ $i_{N}$.

If we are trying to prove that a given directed system $\left\{\mathcal{A}_{*}, i_{*}\right\}$ truly converges to a given $\mathcal{A}_{\infty}$, the third convergence condition is the most critical. Using the notation $i_{N, M}:=i_{M-1} \circ \ldots \circ i_{N}: \mathcal{A}_{N} \hookrightarrow \mathcal{A}_{M}$, an equivalent statement is that $\forall N \forall a, b \in \mathcal{A}_{N}$,

$$
\lim _{m \rightarrow \infty} I_{N+m}\left[i_{N, N+m}(a) i_{N, N+m}(b)\right]=I_{N}(a) I_{N}(b) .
$$

If there are left inverses $I_{N}^{\text {inv }}$ (such that $I_{N}^{\text {inv }} \circ I_{N}=\mathrm{id}$ ), chosen so that the sections $N \mapsto I_{N}^{\text {inv }}(f)$ are continuous, then there is a simpler statement. This convergence 
condition becomes $\forall f_{1}, f_{2} \in \mathcal{A}_{\infty}$

$$
I_{N}\left[I_{N}^{\mathrm{inv}}\left(f_{1}\right) I_{N}^{\mathrm{inv}}\left(f_{2}\right)\right] \underset{N \rightarrow \infty}{\longrightarrow} f_{1} f_{2} .
$$

This is the form used in Section 5.3 .

In this circumstance it is also necessary to check that Vec-lim$\left\{\mathcal{A}_{*}, i_{*}\right\} \subset \mathcal{A}_{\infty}$ really is dense. This means that $I_{N}$ needs to be "asymptotically onto". Using the $I_{N}^{\text {inv's, }}$ this simplifies to the requirement that $\forall f \in \mathcal{A}_{\infty}, I_{N} \circ I_{N}^{\text {inv }}(f) \rightarrow f$ as $N \rightarrow \infty$.

Although this was done for the index set $\mathbb{N}$, it can trivially be generalized to any directed set.

B.2. Direct limit of modules. Given a directed system $\left\{V_{*}, \iota_{*}\right\}$ of finitely generated, projective modules of each $\mathcal{A}_{N}$, we would like to construct a limit module of the limit algebra $\mathcal{A}_{\infty}$. The construction must work in the special case that the system is just $\left\{\mathcal{A}_{*}, i_{*}\right\}$. The vector space direct limit Vec-lim $\left\{V_{*}, \iota_{*}\right\}$ is not itself an $\mathcal{A}_{\infty}$-module; it needs to be completed somehow. Completion is usually done with some norm, but there is generally no natural norm on the $V_{N}$ 's. Instead, complete algebraically.

The algebraic direct product $\Gamma\left(V_{\mathbb{N}}\right)$ is a $\Gamma\left(\mathcal{A}_{\mathbb{N}}\right)$-module, and by restriction an $\mathbb{A}$ module. From the construction of the vector space direct limit, start with the vector space

$$
\stackrel{\circ}{\mathbb{V}}:=\left\{\psi \in \Gamma\left(V_{\mathbb{N}}\right) \mid \exists M \forall N \geq M: \psi_{N+1}=\iota_{N}\left(\psi_{N}\right)\right\} .
$$

Now define $\mathbb{V}$ as the span of $\mathbb{A} \mathbb{V} \subset \Gamma\left(V_{\mathbb{N}}\right)$. I insist that $\mathbb{V}$ be a finitely generated $\mathbb{A}$-module, so there is a convergence condition that any element of $\mathbb{V}$ can be written as the sum of a bounded number of elements of $\mathbb{A} \mathbb{V}$. In other words, $\mathbb{A} \stackrel{\mathbb{V}}{ }+\cdots+\mathbb{A} \mathbb{V}$ stabilizes for some finite number of summands.

It is now easy to construct an $\mathcal{A}_{\infty}$-module. The ideal $\mathbb{A}_{0}$ induces a submodule $\mathbb{A}_{0} \mathbb{V} \subset \mathbb{V}$, and the quotient $V_{\infty}:=\mathbb{V} / \mathbb{A}_{0} \mathbb{V}$ is an $\mathcal{A}_{\infty}$-module. This is the direct limit of modules. Note that its construction requires the map $\mathcal{P}: \mathbb{A} \rightarrow \mathcal{A}_{\infty}$ but does not require any other quantization structure for the algebras.

B.3. Inverse limit of algebras. The limit of the inverse system of algebras is easier to construct. It is

$$
\mathcal{A}_{\infty}=\lim _{\longleftarrow}\left\{\mathcal{A}_{*}, p_{*}\right\}:=\left\{a \in \Gamma_{\mathrm{b}}\left(\mathcal{A}_{\mathbb{N}}\right) \mid \forall N: a_{N-1}=p_{N}\left(a_{N}\right)\right\} .
$$

Again, the $p_{N}$ 's should not be required to be homomorphisms, and again, convergence conditions are necessary.

This limit also inherits a norm $\|a\|:=\lim _{N \rightarrow \infty}\left\|a_{N}\right\|$. This is well defined if the $p_{N}$ 's are required to be norm-contracting. It is then the limit of a nondecreasing sequence that is bounded from above. No additional condition is required to make this nondegenerate since $\|a\| \geq\left\|a_{N}\right\| . \mathcal{A}_{\infty}$ is already complete with respect to this norm.

Since $\mathcal{A}_{\infty}$ consists of sequences of nondecreasing norm, the intersection with $\mathbb{A}_{0}=$ $\Gamma_{0}\left(\mathcal{A}_{\mathbb{N}}\right)$ is 0 . This means that $\mathcal{A}_{\infty}$ injects naturally into $\Gamma_{\mathrm{b}}\left(\mathcal{A}_{\mathbb{N}}\right) / \mathbb{A}_{0}$. Define $\mathbb{A}$ to be the preimage of $\mathcal{A}_{\infty}$ by the quotient homomorphism $\Gamma_{\mathrm{b}}\left(\mathcal{A}_{\mathbb{N}}\right) \rightarrow \Gamma_{\mathrm{b}}\left(\mathcal{A}_{\mathbb{N}}\right) / \mathbb{A}_{0}$; this gives $\mathbb{A}=\mathcal{A}_{\infty}+\mathbb{A}_{0} \subset \Gamma_{\mathrm{b}}\left(\mathcal{A}_{\mathbb{N}}\right)$ 
This $\mathcal{A}_{\infty}$ is also not a priori an algebra. We again need the condition that $\mathcal{A}_{\infty} \subset$ $\Gamma_{\mathrm{b}}\left(\mathcal{A}_{\mathbb{N}}\right) / \mathbb{A}_{0}$ be algebraically closed. This is equivalent to requiring that $\mathbb{A} \subset \Gamma_{\mathrm{b}}\left(\mathcal{A}_{\mathbb{N}}\right)$ be algebraically closed. If $\mathcal{A}_{\infty}$ and $\mathbb{A}$ are algebraically closed, then they are $\mathrm{C}^{*}$-algebras.

For each $N$, there is a canonical surjection $P_{N}: \lim _{\longleftarrow}\left\{\mathcal{A}_{*}, p_{*}\right\} \rightarrow \mathcal{A}_{N}$ which simply takes $a \mapsto a_{N}$. Heuristically $P_{N}=p_{N+1} \circ p_{N+2} \circ \ldots$

This last convergence condition is again the most critical. If we are testing whether $\lim _{\longleftarrow}\left\{\mathcal{A}_{*}, p_{*}\right\} \stackrel{?}{=} \mathcal{A}_{\infty}$, then an equivalent statement is $\forall f_{1}, f_{2} \in \mathcal{A}_{\infty}$,

$$
\lim _{N \rightarrow \infty}\left\|P_{N}\left(f_{1}\right) P_{N}\left(f_{2}\right)-P_{N}\left(f_{1} f_{2}\right)\right\|=0 .
$$

If there are right inverses $P_{N}^{\text {inv }}$ (such that $P_{N} \circ P_{N}^{\text {inv }}=\mathrm{id}$ ), chosen so that $P_{N}^{\text {inv }} \circ$ $P_{N}(f) \rightarrow f$ as $N \rightarrow \infty$, then there is a simpler statement. This convergence condition becomes $\forall f_{1}, f_{2} \in \mathcal{A}_{\infty}$,

$$
P_{N}^{\text {inv }}\left[P_{N}\left(f_{1}\right) P_{N}\left(f_{2}\right)\right] \underset{N \rightarrow \infty}{\longrightarrow} f_{1} f_{2} .
$$

This is the form used in Section 5.3 .

B.4. Inverse limit of modules. This construction is very much the same as in B.2 for a direct limit of modules. For an inverse system $\left\{V_{*}, \pi_{*}\right\}$ of modules, first construct the vector space

$$
\stackrel{\circ}{\mathbb{V}}:=\left\{\psi \in \Gamma\left(V_{\mathbb{N}}\right) \mid \forall N: \psi_{N-1}=p_{N}\left(\psi_{N}\right)\right\} .
$$

Again define $\mathbb{V}$ as the span of $\mathbb{A} \mathbb{V}$, and the convergence condition is that $\mathbb{A} \mathbb{V}+\cdots+\mathbb{A} \mathbb{V}$ stabilizes for some finite number of summands.

Define $\lim _{\longleftarrow}\left\{V_{*}, \pi_{*}\right\}:=\mathbb{A} / \mathbb{A}_{0} \mathbb{V}$.

\section{Appendix C. Review of Representation Theory}

Let $G$ be a compact, simply connected, semisimple Lie group. This always contains a Cartan subgroup $T$. This is a maximal abelian subgroup which is always of the form $\mathrm{U}(1)^{\ell}$ (a torus group). Any two Cartan subgroups of $G$ are conjugate, so it is irrelevant which one we now fix and call the Cartan subgroup. Since the irreducible representations of $\mathrm{U}(1)$ are one-dimensional and classified by $\mathbb{Z}$, the irreducible representations of $T$ are one-dimensional and classified by the lattice $\mathbb{Z}^{\ell}$.

The Cartan subalgebra $\mathfrak{C} \subset \mathfrak{g}$ is the Lie algebra of $T$. Any vector in an irreducible representation of $T$ is an eigenvector of any element of $\mathfrak{C}$; the eigenvalue depends linearly on the position of the representation in the above lattice (and on the element of $\mathfrak{C}$ ). The lattice is therefore naturally thought of as lying in the dual $\mathfrak{C}^{*}$ of the Cartan subalgebra. It is called the weight lattice. There is a natural inner product (the Cartan-Killing form) on the Lie algebra $\mathfrak{g}$; using this, there is a natural sense in which $\mathfrak{C}^{*} \subset \mathfrak{g}^{*}$.

There are some symmetries to $\mathfrak{C}^{*}$, residual from the action of $G$ on $\mathfrak{g}^{*}$. The symmetry group of $\mathfrak{C}^{*}$ is the subgroup of $G$ that preserves $\mathfrak{C}^{*} \subset \mathfrak{g}^{*}$, modulo the subgroup that acts trivially on $\mathfrak{C}^{*}$. This is called the Weyl group $W$ and is finite. Since both are naturally constructed from the pair $\mathfrak{C} \subset \mathfrak{g}$, the Weyl group preserves the weight 
lattice. The Weyl group is generated by a set of reflections across hyperplanes in $\mathfrak{C}^{*}$. These plains divide $\mathfrak{C}^{*}$ into wedges called Weyl chambers; each Weyl chamber is a fundamental domain of the $W$ action on $\mathfrak{C}^{*}$, this means that the $W$-orbit of any point of $\mathfrak{C}^{*}$ intersects a given closed Weyl chamber at least once and intersects the interior of a given Weyl chamber at most once.

We can choose a basis of the weight lattice; that is, a set of fundamental weights $\left\{\pi_{j}\right\}$ such that the weight lattice is the integer span $\sum_{j} \mathbb{Z} \pi_{j}$. Given the choice of $\mathfrak{C}$, the fundamental weights are unique modulo the freedom to change their signs. Fix a set of fundamental weights. The natural index set for the fundamental weights is the set of vertices of the Dynkin diagram of $\mathfrak{g}$. The positive span of the fundamental weights $\sum_{j} \mathbb{R}_{+} \pi_{j}$ is precisely a (closed) Weyl chamber. Call this the positive Weyl chamber $\mathcal{C}_{+}$. The weights that lie in $\mathcal{C}_{+}$are nonnegative integer combinations of the fundamental weights and are called dominant weights. Since it is a fundamental domain of the $W$ action, the positive Weyl chamber $\mathcal{C}_{+}$can naturally be identified with $\mathfrak{C}^{*} / W$.

Given an irreducible representation of $G$, it can also be regarded as a $T$ representation. The representation space therefore naturally decomposes into a direct sum of subspaces associated with different weights. The set of weights that occur is $W$ invariant. The subspace associated with the dominant weight furthest from 0 is always 1-dimensional; that weight is called the highest weight of the representation. Nonisomorphic irreducible representations have distinct highest weights and any dominant weight is the highest weight of some representation. The irreducible representations of $G$ are therefore exactly classified by dominant weights. I denote the representation space with highest weight $\lambda$ as $(\lambda)$.

Weights are additive under the tensor product. If two vectors have weights $\lambda$ and $\mu$, then their tensor product has weight $\lambda+\mu$. Because of this, the highest weight of the (reducible) representation $(\lambda) \otimes(\mu)$ is $\lambda+\mu$. The decomposition of $(\lambda) \otimes(\mu)$ into irreducibles will therefore always contain precisely one copy of $(\lambda+\mu)$; this irreducible representation is called the Cartan product of $(\lambda)$ and $(\mu)$.

For each irreducible representation, we can choose a normalized vector $\Psi^{\lambda} \in(\lambda)$ in the highest weight subspace. This is called a highest weight vector. Their phases are arbitrary, but can be chosen consistently so that $\Psi^{\lambda} \otimes \Psi^{\mu}=\Psi^{\lambda+\mu} \in(\lambda+\mu) \subset(\lambda) \otimes(\mu)$.

The linear dual of an irreducible representation is also an irreducible representation; we can therefore define $\lambda^{*}$ by the property $\left(\lambda^{*}\right)=(\lambda)^{*}$. This is a linear transformation on the weight lattice; it simply permutes the fundamental weights and is given by an automorphism (possibly trivial) of the Dynkin diagram.

Whenever $\lambda-\mu$ is a dominant weight, $(\lambda) \otimes\left(\mu^{*}\right)$ will contain precisely one copy of $(\lambda-\mu)$. In particular, if $\lambda=\mu$ this says that $(\lambda) \otimes\left(\lambda^{*}\right)$ contains one copy of the trivial representation; this is little more than the definition of the dual. 


\section{Appendix D. Coadjoint Orbits}

The purpose of this appendix is to describe the rationale for restricting attention to coadjoint orbits, and then to discuss some of the structure of coadjoint orbits. Toward this goal, I first discuss a more general structure:

D.1. Symplectic structure. Thus far I have entirely avoided mentioning something which is usually mentioned first in discussions of quantization - the symplectic structure.

Assume $\mathcal{M}$ to be a manifold. Suppose that part of our quantization structure is a system of maps $I_{N}: \mathcal{A}_{N} \hookrightarrow \mathcal{C}(\mathcal{M})$, identifying quantum operators to classical functions. We can choose a system of right inverses; that is, maps $I_{N}^{\text {inv }}: \mathcal{C}(\mathcal{M}) \rightarrow \mathcal{A}_{N}$ such that $I_{N} \circ I_{N}^{\text {inv }}$ is the identity map $\mathcal{A}_{N} \rightarrow \mathcal{A}_{N}$. Using these, we can pull back the products on each of the $\mathcal{A}_{N}$ 's to $\mathcal{C}(\mathcal{M})$, giving a sequence of products

$$
f *^{N} f^{\prime}=I_{N}\left[I_{N}^{\text {inv }}(f) I_{N}^{\text {inv }}\left(f^{\prime}\right)\right] .
$$

By construction, these converge to the ordinary product of functions as $N \rightarrow \infty$.

Suppose that the quantization is compatible with the smooth structure of $\mathcal{M}$ in the sense that for smooth functions $f, f^{\prime} \in \mathcal{C}^{\infty}(\mathcal{M})$ the correction $f *^{N} f^{\prime}-f f^{\prime}$ is of order 9 $\frac{1}{N}$. I will assume that any quantization of interest satisfies this. This compatibility means that the function

$$
\left\{f, f^{\prime}\right\}:=\lim _{N \rightarrow \infty}-i N\left(f *^{N} f^{\prime}-f^{\prime} *^{N} f\right)
$$

is well defined. This is the Poisson bracket of $f$ and $f^{\prime}$; it is easily seen to be, by construction, antisymmetric and a derivation in both arguments. This means that there exists an antisymmetric, contravariant, rank-2 tensor $\square \pi^{i j}$ such that the Poisson bracket is given by $\left\{f, f^{\prime}\right\}=\left\langle\pi, d f \wedge d f^{\prime}\right\rangle \equiv \pi^{i j} d f_{i} d f_{j}^{\prime}$.

With the assumption that the algebras $\mathcal{A}_{N}$ are finite-dimensional, the $\pi$ should be nondegenerate if thought of as a map from 1-forms to tangent vectors. This means that it has an inverse $\omega=\pi^{-1}$, which is naturally a 2-form. The Poisson bracket also satisfies the Jacobi identity, and this implies that $\omega$ is a closed 2 -form $(d \omega=0)$. This $\omega$ is the symplectic form. Although right inverses are not unique, the Poisson bracket and symplectic form are independent of the specific choice of the $I_{N}^{\mathrm{inv}}$ 's here.

D.2. Why coadjoint orbits? Let $\mathcal{M}$ be a compact manifold and assume that a compact, semisimple Lie group $G$ acts smoothly and transitively on $\mathcal{M}$. This implies that $\pi_{1}(\mathcal{M})$ is finite, and thus $H^{1}(\mathcal{M} ; \mathbb{R})=0$. Everything we do should be $G$ equivariant.

Because $G$ acts smoothly on $\mathcal{M}$, the elements of the Lie algebra $\mathfrak{g}$ of $G$ define certain vector fields on $\mathcal{M}$. Since the quantization is assumed to be $G$-equivariant,

\footnotetext{
${ }^{9}$ This can be generalized slightly by replacing $\frac{1}{N}$ with some other function $\hbar(N)$ that goes to 0 as $N \rightarrow \infty$. The implication (existence of Poisson bracket) remains the same.

${ }^{10}$ This is also called a bivector.
} 
the symplectic form must be $G$-invariant. This implies that for any $\xi \in \mathfrak{g}$ thought of as a vector field on $\mathcal{M}$,

$$
\left.\left.\left.0=\mathcal{L}_{\xi} \omega=d(\xi\lrcorner \omega\right)+\xi\right\lrcorner d \omega=d(\xi\lrcorner \omega\right) ;
$$

so (using $H^{1}=0$ ) there is a "Hamiltonian" $h(\xi) \in \mathcal{C}^{\infty}(\mathcal{M})$ such that $\left.\xi\right\lrcorner \omega=d h(\xi)$, which is well defined modulo constants. The constant can be fixed by requiring that the average of $h(\xi)$ over $\mathcal{M}$ is 0 . This gives a well-defined linear map $h: \mathfrak{g} \rightarrow \mathcal{C}(\mathcal{M})$. For any $x \in \mathcal{M}$, the evaluation $\xi \mapsto h(\xi)(x)$ is a linear map $\mathfrak{g} \rightarrow \mathbb{C}$; in other words, $h$ lets us map $x$ into the linear dual $\mathfrak{g}^{*}$. That map is the "moment map" $\Phi: \mathcal{M} \rightarrow \mathfrak{g}^{*}$ (see [13]).

Because $\mathcal{M}$ is homogeneous and compact, the moment map turns out to be an embedding, so effectively $\mathcal{M} \subset \mathfrak{g}^{*}$. By transitivity, $\mathcal{M}$ is precisely the orbit of any of its points under the natural "coadjoint" action $\operatorname{Ad}_{G}^{*}$ of $G$ on $\mathfrak{g}^{*}$, so any of the homogeneous spaces we are considering is a coadjoint orbit.

Since $\mathfrak{g}^{* *}=\mathfrak{g}$, any element of $\mathfrak{g}$ is naturally thought of as a linear function on $\mathfrak{g}^{*}$. The Lie bracket on these of course satisfies the Jacobi identity, and extends to a unique Poisson bracket for all functions on $\mathfrak{g}^{*}$. If $x_{i}$ are linear coordinates on $\mathfrak{g}^{*}$ then the Poisson bivector $\pi$ on $\mathfrak{g}^{*}$ is given by $\pi_{i j}=C_{i j}^{k} x_{k}$. This Poisson bivector is degenerate, but restricts to a nondegenerate one on any coadjoint orbit. This makes any coadjoint orbit symplectic. The set of homogeneous spaces we are interested in is therefore precisely the set of coadjoint orbits of compact Lie groups.

The single point $\{0\} \subset \mathfrak{g}^{*}$ is trivially a coadjoint orbit. It is an exception to some of the statements in this paper, but an utterly uninteresting one, so I will not mention it again.

D.3. Structure of coadjoint orbits. We are interested in all coadjoint orbits, but all coadjoint orbits intersect $\mathfrak{C}^{*} \subset \mathfrak{g}^{*}$, so it is sufficient to consider the orbits of all $\Lambda \in \mathfrak{C}^{*}$. These are still not all distinct; $\mathcal{O}_{\Lambda}=\mathcal{O}_{\Lambda^{\prime}}$ if (and only if) $\Lambda$ and $\Lambda^{\prime}$ are mapped to one another by the Weyl group $W$. The set of distinct coadjoint orbits is therefore $\mathfrak{g}^{*} / G \cong \mathfrak{C}^{*} / W \cong \mathcal{C}_{+}$, using the fact that the Weyl chamber $\mathcal{C}_{+}$is a fundamental domain of the $W$ action (App. C).

We would like to express the coadjoint orbit $\mathcal{O}_{\Lambda}$ of $\Lambda \in \mathcal{C}_{+}$as $G / H$. So what is $H$ ? It is the subgroup of $G$ leaving $\Lambda$ invariant, or equivalently the centralizer

$$
H=Z_{\Lambda}(G) \equiv\left\{h \in G \mid \operatorname{Ad}_{h}(\Lambda)=\Lambda\right\}
$$

if $\Lambda$ is identified to an element of $\mathfrak{g}$ using the inner product. In this sense, $\Lambda \in \mathfrak{C}$, so because $\mathfrak{C}$ is Abelian, $\mathfrak{C} \subset \mathfrak{h}$. This implies that the Cartan subgroup $T$ is a subgroup of $H$, so $T$ can be used as the Cartan subgroup of $H$, and weights of $G$ and $H$ are naturally identified. There are, however, weights which are dominant for $H$ that are not for $G$, and the Weyl groups are different.

Expand $\Lambda$ in the basis $\left\{\pi_{j}\right\}$, and mark the vertices $j \in \operatorname{Dynkin}(\mathfrak{g})$ for which $\pi_{j}$ has a nonzero coefficient in $\Lambda$. The vertices of the Dynkin diagram are also the natural index set for the dual basis of fundamental roots. In the standard root decomposition of $\mathfrak{g}^{\mathbb{C}}, E_{\alpha}$ commutes with $\Lambda$ and is thus in $\mathfrak{h}^{\mathbb{C}}$ if and only if $\alpha$ is orthogonal to $\Lambda$. This is true precisely if, in the expansion in fundamental roots, $\alpha$ has 0 coefficients for all 
Coadjoint Orbits with $\operatorname{dim} \leq 6$

\begin{tabular}{|ccccc|}
\hline dim. & Name & $\mathcal{O}_{\Lambda}$ & $G / H$ & Diagram \\
\hline 2 & Sphere & $S^{2}$ & $\mathrm{SU}(2) / \mathrm{U}(1)$ & $\bullet$ \\
4 & Complex projective space & $\mathbb{C P}^{2}$ & $\mathrm{SU}(3) / \mathrm{U}(2)$ & $\bullet$ \\
6 & Complex projective space & $\mathbb{C P}^{3}$ & $\mathrm{SU}(4) / \mathrm{U}(3)$ & $\bullet$ \\
& $"$ & $"$ & $\mathrm{Sp}(4) / \mathrm{U}(1) \times \mathrm{Sp}(2)$ & $\Longrightarrow$ \\
6 & Complex flag variety & & $\mathrm{SU}(3) / \mathrm{U}(1) \times \mathrm{U}(1)$ & $\bullet \bullet$ \\
6 & Double cover of real Grassmanian & $\widetilde{G}_{2,5}^{\mathbb{R}}$ & $\mathrm{SO}(5) / \mathrm{SO}(2) \times \mathrm{SO}(3)$ & $\bullet 0$ \\
\hline
\end{tabular}

the marked vertices of $\operatorname{Dynkin}(\mathfrak{g})$. This means that $\mathfrak{h}^{\mathbb{C}}$ is spanned by $\mathfrak{C}$ and the $E_{\alpha}$ 's that are supported on the unmarked vertices.

This gives a simple, diagrammatic way of calculating $\mathfrak{h}$ : The Lie algebra $\mathfrak{h}$ of $H$ is the sum of a copy of $\mathfrak{u}(1)$ for every marked vertex and the Lie algebra of whatever Dynkin diagram is left after deleting all the marked vertices (and adjoining edges).

(This diagrammatic method is also described in [2], the only difference is that the complementary set of vertices is marked.)

This shows that, up to homeomorphism, the orbit $\mathcal{O}_{\Lambda}$ depends only on which coefficients are nonzero. On the other hand the symplectic structure and metric do vary with $\Lambda$. Since the number of marked vertices is the number of nonzero coefficients for $\Lambda$, this is the number of parameters that orbits in a given homeomorphism class vary by. One of these degrees of freedom simply corresponds to rescaling.

I use the notation $[\lambda]$ for the irreducible $H$-representation with highest weight $\lambda$. If the weight $\lambda$ is a combination of fundamental weights corresponding to marked vertices of $\operatorname{Dynkin}(\mathfrak{g})$, then the semisimple part of $\mathfrak{h}$ acts trivially on $[\lambda]$. In this case $[\lambda]$ is one-dimensional and is just a representation of the abelian part of $\mathfrak{h}$. The weights $N \Lambda$ are of this type.

In general, if $[\lambda]$ is one-dimensional then $[\lambda]^{*}=[-\lambda]$. If $[\lambda]$ is one-dimensional and $\mu$ is arbitrary, then $[\lambda] \otimes[\mu]=[\lambda+\mu]$.

D.4. Examples. The existence of the symplectic structure implies that a coadjoint orbit must be even-dimensional. The table lists the lowest-dimensional coadjoint orbits (all those with dimension $\leq 6$ ). Note that $\mathbb{C} P^{3}$ occurs in two forms.

A less trivial example is given by the diagram $\bullet-\cdots 0$. In this case $G=$ $\widetilde{\mathrm{SO}}(11)$, and (modulo coverings) $H \approx \mathrm{U}(1) \times \mathrm{SU}(2) \times \mathrm{U}(1) \times \mathrm{SO}(5)$. The dimension is $\operatorname{dim} G-\operatorname{dim} H=55-(1+3+1+10)=40$.

Notably, $S^{2}$ is the only sphere which is a coadjoint orbit. In fact it is the only sphere which admits a symplectic structure, equivariant or not. This is because the symplectic form on a compact manifold always has a nontrivial cohomology class, implying $H^{2}(\mathcal{M}) \neq 0$. The 2 -sphere is the only sphere such that $H^{2}\left(S^{n}\right) \neq 0$. This means that with the reasonable seeming condition of respecting the smooth structure (as described in D.1), no other sphere may be quantized. For a claim to the contrary, see [11]. 


\section{Appendix E. Projective space}

There is a (very) slightly different perspective on how the formula (5.5) for the injection $I_{N}: \mathcal{A}_{N} \hookrightarrow \mathcal{C}\left(\mathcal{O}_{\Lambda}\right)$ comes about. It can be thought of as resulting from a natural embedding of $\mathcal{O}_{\Lambda}$ into the projectivisation $\mathbb{P}(N \Lambda)$ of the representation $(N \Lambda)$. The idea is simply that since $\Psi^{N \Lambda}$ is fixed modulo phase by $H$, its projective equivalence class $\left[\Psi^{N \Lambda}\right] \in \mathbb{P}(N \Lambda)$ is exactly fixed by $H$. Indeed $H$ is the entire isotropy group of this point. This means that the equivariant map that takes $\mathcal{O}_{\Lambda} \ni o \mapsto\left[\Psi^{N \Lambda}\right]$ is an embedding $\mathcal{O}_{\Lambda} \hookrightarrow \mathbb{P}(N \Lambda)$.

Any point $[\psi] \in \mathbb{P}(N \Lambda)$ determines a state (a normalized element of the dual) of $\mathcal{A}_{N}$. This takes

$$
a \mapsto \frac{\langle\psi|a| \psi\rangle}{\langle\psi \mid \psi\rangle} .
$$

So, we can naturally map $\mathcal{O}_{\Lambda} \hookrightarrow \mathbb{P}(N \Lambda) \rightarrow \mathcal{A}_{N}^{*}$. From this point the story continues in the same way as in Section 5.2.

Suppose that $|x\rangle,|y\rangle$, and $|z\rangle$ are (unnormalized) vectors in $(\Lambda)$ such that $x \mapsto$ $[|x\rangle] \in \mathbb{P}(\Lambda)$, et cetera. The formula (5.12) for $K_{1}$ can be rewritten as

$$
[\operatorname{dim}(\Lambda)]^{-2} K_{1}(x, y, z)=\frac{\langle x \mid y\rangle\langle y \mid z\rangle\langle z \mid x\rangle}{\langle x \mid x\rangle\langle y \mid y\rangle\langle z \mid z\rangle} .
$$

A continuous choice of these vectors cannot be made globally, but it can be made in a small neighborhood of $o$. As in Section 5.3, let's fix $x=o$. The obvious choice for $|o\rangle$ is $\left|\Psi^{\Lambda}\right\rangle$. The arbitrariness in the other vectors is the freedom to multiply by a scalar. If we to fix these vectors by letting $\left\langle\Psi^{\Lambda} \mid y\right\rangle=1$ (and likewise for $z$ ), then (E.2) simplifies to

$$
[\operatorname{dim}(\Lambda)]^{-2} K_{1}(o, y, z)=\frac{\left\langle\Psi^{\Lambda} \mid y\right\rangle\langle y \mid z\rangle\left\langle z \mid \Psi^{\Lambda}\right\rangle}{\left\langle\Psi^{\Lambda} \mid \Psi^{\Lambda}\right\rangle\langle y \mid y\rangle\langle z \mid z\rangle}=\frac{\langle y \mid z\rangle}{\langle y \mid y\rangle\langle z \mid z\rangle} .
$$

Now suppose that we have a complex coordinate system for $y$ and $z$, that the coordinates are vectors $v$ and $\zeta$ in a subspace of $(\Lambda)$, and that to first order $|y\rangle$ is given by

$$
|y\rangle \approx\left|\Psi^{\Lambda}\right\rangle+|v\rangle
$$

(and $|z\rangle$ is given by $\zeta$ ). From this, the inner product $\langle y \mid z\rangle$ can be calculated to second order

$$
\begin{aligned}
\langle y \mid z\rangle & =1+\left\langle y \mid z-\Psi^{\Lambda}\right\rangle \\
& =1+\left\langle y-\Psi^{\Lambda} \mid z-\Psi^{\Lambda}\right\rangle \\
& \approx 1+\langle v \mid \zeta\rangle .
\end{aligned}
$$

Inserting this into $(\mathbb{E} .3)$ gives a formula for $K_{1}$ to second order

$$
\begin{aligned}
{[\operatorname{dim}(\Lambda)]^{-2} K_{1}(o, y, z) } & \approx \frac{1+\langle v \mid \zeta\rangle}{\left(1+\|v\|^{2}\right)\left(1+\|\zeta\|^{2}\right)} \\
& \approx 1-\|v\|^{2}-\|\zeta\|^{2}+\langle v \mid \zeta\rangle .
\end{aligned}
$$




\section{Notation}

$[\cdot, \cdot]_{-}$Commutator, $[a, b]_{-}=a b-b a$. (5.1).

$\mathcal{A}_{\hat{\mathcal{I}}}$ The bundle of algebras over $\hat{\mathcal{I}}$. Sec. 2 .

$\mathcal{A}_{N}$ Quantum algebra at index $N \in \mathcal{I}$, later $\operatorname{End}(N \Lambda)$. Sec's 2, 5 .

$\mathbb{A} \quad \mathrm{C}^{*}$-algebra of continuous sections of $\mathcal{A}_{\hat{\mathcal{I}}}$. Sec's 2 , 5.1, B.1, B.3.

$\AA$ Preliminary vector space, dense in $\mathbb{A}$. (B.2).

$\mathbb{A}_{0}=\Gamma_{0}\left(\mathcal{A}_{\mathcal{I}}\right)$, an ideal in $\mathbb{A}$, the Kernel of $\mathcal{P}: \mathbb{A} \rightarrow \mathcal{A}_{\infty}$. Sec's 5.1, B.1, B.3.

$\mathcal{C}_{n}$ The $n$ 'th Casimir polynomial. (5.2).

$c_{n}(N \Lambda)$ The eigenvalue of the Casimir operator $\mathcal{C}_{n}(J)$ acting on $(N \Lambda)$. (5.2).

$\mathfrak{C}$ Cartan subalgebra of $\mathfrak{g}$. App. $\mathrm{C}$.

$\mathcal{C}_{+}$Positive Weyl chamber in $\mathfrak{C}^{*}$. Sec's 4.1, C.

$e_{N} \quad$ The function $e_{N}: \mathcal{O}_{\Lambda} \hookrightarrow \mathcal{A}_{N}, g H \mapsto\left|g \Psi^{N \Lambda}\right\rangle\left\langle g \Psi^{N \Lambda}\right|$. 5.7)

End Endomorphisms, the algebra of matrices on some vector space. Sec. 5 .

$\epsilon$ Volume form on $\mathcal{O}_{\Lambda}$, normalized so that $\int_{\mathcal{O}_{\Lambda}} \epsilon=1$. (5.8).

$\Gamma$ The space of continuous sections of a bundle. App. A.

$\Gamma_{\mathrm{b}}$ The space of norm-bounded sections. App. A.

$\Gamma_{0}$ The space of continuous sections vanishing at $\infty$. App. A.

$\Gamma_{\mathrm{c}}$ The space of compactly supported sections. App. A.

$\Gamma_{\text {poly }}$ The space of polynomial sections. Sec. 8 .

$\mathcal{I}$ Index set of the quantization. Sec. 2 .

$\hat{\mathcal{I}}=\mathcal{I} \cup\{\infty\}$. Sec. 2 .

$J_{i}$ The basis of Hermitian generators of $\mathfrak{g}$. Sec. 5.1.

$\mathbb{N}=\{1,2, \ldots\}$.

$\hat{\mathbb{N}}=\{1,2, \ldots, \infty\}$.

( $\lambda$ ) $G$-representation space with highest weight $\lambda$. Sec's 5, C.

[ $\lambda] H$-representation space with highest weight $\lambda$. Sec's 6.3 , D.3.

$\lambda^{*} \quad$ Weight vector such that $\left(\lambda^{*}\right)=(\lambda)^{*}$. Sec's 5.2, 9.

$\mathcal{O}_{\Lambda}$ Coadjoint orbit passing through the weight vector $\Lambda \in \mathcal{C}_{+} \subset \mathfrak{g}^{*}$. Sec. 4.1.

$\mathcal{P}$ The surjection $\mathbb{A} \rightarrow \mathcal{C}(\mathcal{M})$. Sec. 2.

$\mathbb{P}$ Projectivization of a vector space. App. $\mathbb{E}$.

$\Pi$ Projection onto some subrepresentation. Sec's 5.2, 6.2.

$\Psi^{\lambda} \quad$ Normalized highest weight vector in $(\lambda)$. Sec's 5.2, $\mathbf{9}$.

$Q_{N}^{\lambda} \quad$ Projection such that $V_{N}^{\lambda}=\left[\mathcal{A}_{N} \otimes(\mu)\right] Q_{N}$. Sec. 6.1

$\widetilde{\operatorname{tr}}$ Trace normalized so that $\widetilde{\operatorname{tr}} 1=1$. Sec. 5.2.

$V_{N}$ Module of the algebra $\mathcal{A}_{N}$ in the quantization of the bundle $V$. Sec. 3 .

$V_{\infty}=\Gamma(V)$, which is a module of $\mathcal{C}(\mathcal{M})$. Sec. 3 .

$V_{\mathbb{N}}^{\lambda} \quad$ The bundle of quantum modules associated to the $G$-weight $\lambda$. Sec. 6.1.

$\mathbb{V}$ A-module expressing a quantization of $V$. Sec. 3 .

$\mathbb{V}$ Vector space which generates $\mathbb{V}$ as an $\mathbb{A}$-module. App's B.2, B.4.

Acknowledgments. I wish to thank Ranee Brylinski and Nigel Higson for extensive discussions. This material is based upon work supported under a National Science 
Foundation Graduate Fellowship. Also supported in part by NSF grant PHY95-14240 and by the Eberly Research Fund of the Pennsylvania State University

\section{REFERENCES}

[1] Berezin, F. A.: General Concept of Quantization. Commun. Math. Phys. 40, 153-174 (1975).

[2] Bordemann, M., Forger, M., Römer, H.: Homogeneous Kähler Manifolds: Paving the Way Towards New Supersymmetric Sigma Models. Commun. Math. Phys. 102, 605-647 (1986).

[3] Chamseddine, A., Connes, A.: The Spectral Action Principle. E-print, hep-th/9606001. Commun. Math. Phys. 186, 73-750 (1997).

[4] Connes, A.: Noncommutative Geometry. New York: Academic Press, 1994.

[5] _ Gravity Coupled with Matter and the Foundation of Noncommutative Geometry. Eprint, hep-th/9603053. Commun. Math. Phys. 182, 155-176 (1996).

[6] Dixmier, J.: C*-algebras. Amsterdam: North Holland, 1982.

[7] Fillmore, P. A.: A User's Guide to Operator Algebras. New York: Wiley Interscience, 1996.

[8] Grosse, H., Klimčík, C., Prešnajder, P.: Towards Finite Quantum Field Theory in Noncommutative Geometry. E-print, hep-th/9505175. Int. J. Theor. Phys. 35, 231-244 (1996).

[9] _ : Field Theory on a Supersymmetric Lattice. E-print, hep-th/9507074. Commun. Math. Phys. 185, 155-175 (1997).

[10] _ : Topologically Nontrivial Field Configurations in Noncommutative Geometry. E-print, hep-th/9510083. Commun. Math. Phys. 178, 507-526 (1996).

[11] : On Finite 4-D Quantum Field Theory in Noncommutative Geometry. E-print, hep-th/9602115. Commun. Math. Phys. 180, 429-438 (1996).

[12] Grosse, H., Prešnajder, P.: The Construction of Noncommutative Manifolds Using Coherent States. Lett. Math. Phys. 28, 239-250 (1993).

[13] Guillemin, V., Sternberg, S.: Symplectic Techniques in Physics. Cambridge: Cambridge University Press, 1984.

[14] Hawkins, E.: Geometric Quantization of Vector Bundles. E-print, math.QA/9808116.

[15] Helgason, S.: Differential geometry, Lie groups, and Symmetric Spaces. Pure and Applied Mathematics, volume 80. New York: Academic Press, 1978.

[16] LAnce, E. C.: Hilbert $C^{*}$-modules. London Mathematical Society Lecture Note Series, no. 210. Cambridge: Cambridge University Press, 1995.

[17] Onishchick, A. L., Vinberg, E. B.: Lie Groups and Algebraic Groups. New York: Springer-Verlag, 1988.

[18] Perelemov, A.: Generalized Coherent States and their Applications. Berlin: SpringerVerlag, 1986.

[19] Rieffel, M. A.: Quantization and $C^{*}$-algebras. $C^{*}$-algebras 1943-1993: a 50 Year Celebration (R. DorAn ed.). Contemp. Math. 167, 66-97 (1994).

[20] Schwartz, J. T.: Differential Geometry and Topology. New York: Gordon and Breach, 1968.

[21] Wegge-Olsen, N. E.: K-theory and C*-algebras. Oxford: Oxford University Press, 1993. 